Article

\title{
On Extended General Mittag-Leffler Functions and Certain Inequalities
}

\author{
Marcela V. Mihai ${ }^{1}$, Muhammad Uzair Awan ${ }^{2, *}$, Muhammad Aslam Noor ${ }^{3}{ }^{\circledR}$, Tingsong Du ${ }^{4}(\mathbb{D}$, \\ Artion Kashuri ${ }^{5}$ and Khalida Inayat Noor ${ }^{3}$ (iD \\ 1 Department Scientific-Methodical Sessions, Romanian Mathematical Society-Branch Bucharest, \\ Academy Street no. 14, RO-010014 Bucharest, Romania; marcelamihai58@yahoo.com \\ 2 Department of Mathematics, Government College University, Faisalabad, Punjab 38000, Pakistan \\ 3 Department of Mathematics, COMSATS University Islamabad, Islamabad 44000, Pakistan; \\ noormaslam@gmail.com (M.A.N.); khalidan@gmail.com (K.I.N.) \\ 4 Department of Mathematics, College of Science, China Three Gorges University, Yichang 443002, China; \\ tingsongdu@ctgu.edu.cn \\ 5 Department of Mathematics, Faculty of Technical Science, University “Ismail Qemali”, 9401 Vlora, Albania; \\ artionkashuri@gmail.com \\ * Correspondence: awan.uzair@gmail.com
}

Received: 8 May 2019; Accepted: 16 June 2019; Published: 18 June 2019 updates

\begin{abstract}
In this paper, we introduce and investigate generalized fractional integral operators containing the new generalized Mittag-Leffler function of two variables. We establish several new refinements of Hermite-Hadamard-like inequalities via co-ordinated convex functions.
\end{abstract}

Keywords: co-ordinated convex function; Hermite-Hadamard inequalities; Mittag-Leffler function

MSC: 26D15; 26A51; 26A33; 33E12

\section{Introduction and Preliminaries}

The Hermite-Hadamard inequality states that if a function $\Psi: I \subseteq \mathbb{R} \rightarrow \mathbb{R}$ is convex, then

$$
\Psi\left(\frac{a+b}{2}\right) \leq \frac{1}{b-a} \int_{a}^{b} \Psi(x) \mathrm{d} x \leq \frac{\Psi(a)+\Psi(b)}{2},
$$

where $a, b \in I$ with $a<b$. Both inequalities hold in the reversed direction if $\Psi$ is concave.

In recent years, many researchers have turned their attention to the Hermite-Hadamard inequality and have found many variations and generalizations of it via various types of convexity. Some of this research is related to functions that are convex on the coordinates (see, for instance, [1-5] and the references therein).

Coordinated convex functions are defined as:

Definition 1 ([3]). Let us consider the bidimensional interval $\Delta=[a, b] \times[c, d]$ in $\mathbb{R}^{2}$ with $a<b, c<d$. A function $\Psi: \Delta \rightarrow \mathbb{R}$ will be called convex on the coordinates if the partial mappings $\Psi_{y}:[a, b] \rightarrow \mathbb{R}$, $\Psi_{y}(u)=\Psi(u, y)$ and $\Psi_{x}:[c, d] \rightarrow \mathbb{R}, \Psi_{x}(v)=\Psi(x, v)$ are convex where defined for all $y \in[c, d]$, and $x \in[a, b]$. Recall mapping $\Psi: \Delta \rightarrow \mathbb{R}$ is convex on the coordinates on $\Delta$ if the following inequality holds:

$$
\begin{aligned}
\Psi(t x+(1-t) y, s u+(1-s) w) & \leq t s \Psi(x, u)+t(1-s) \Psi(x, w) \\
& +s(1-t) \Psi(y, u)+(1-t)(1-s) \Psi(y, w)
\end{aligned}
$$

for all $(x, u),(y, w) \in \Delta$ and $t, s \in[0,1]$. 
Discuss some preliminaries of fractional calculus.

Definition 2. Let $\Psi \in L[a, b]$, where $a \geq 0$. The Riemann-Liouville integrals $J_{a+}^{v} \Psi$ and $J_{b-}^{v} \Psi$, of order $v>0$, are defined by

$$
J_{a+}^{v} \Psi(x)=\frac{1}{\Gamma(v)} \int_{a}^{x}(x-t)^{v-1} \Psi(t) \mathrm{d} t, \text { for } x>a
$$

and

$$
J_{b-}^{v} \Psi(x)=\frac{1}{\Gamma(v)} \int_{x}^{b}(t-x)^{v-1} \Psi(t) \mathrm{d} t, \text { for } x<b,
$$

respectively. Here, $\Gamma(v)=\int_{0}^{\infty} e^{-t} t^{v-1} \mathrm{~d} t$ is the gamma function. We also make the convention

$$
J_{a+}^{0} \Psi(x)=J_{b-}^{0} \Psi(x)=\Psi(x) .
$$

More details about the Riemann-Liouville fractional integrals may be found in [6].

Salim and Faraj [7] have defined the generalized fractional integral operators containing Mittag-Leffler functions:

Definition 3. Let $\mu, v, k, l, \gamma$ be positive real numbers and $\omega \in \mathbb{R}$. Then the generalized fractional integral operators containing Mittag-Leffler function $\varepsilon_{\mu, v, l, \omega, a^{+}}^{\gamma, \delta, k}$ and $\varepsilon_{\mu, v, l, \omega, b^{-}}^{\gamma, \delta, k}$ a real-valued continuous function $\Psi$ are defined by:

$$
\left(\varepsilon_{\mu, v, l, \omega, a^{+}}^{\gamma, \delta, k} \Psi\right)(x)=\int_{a}^{x}(x-t)^{v-1} E_{\mu, v, l}^{\gamma, \delta, k}\left(\omega(x-t)^{\mu}\right) \Psi(t) \mathrm{d} t
$$

and

$$
\left(\varepsilon_{\mu, v, l, \omega, b^{-}}^{\gamma, \delta, k} \Psi\right)(x)=\int_{x}^{b}(t-x)^{v-1} E_{\mu, v, l}^{\gamma, \delta, k}\left(\omega(t-x)^{\mu}\right) \Psi(t) \mathrm{d} t,
$$

respectively, where the function $E_{\mu, v, l}^{\gamma, \delta, k}$ is a generalized Mittag-Leffler function defined as

$$
E_{\mu, v, l}^{\gamma, \delta, k}(t)=\sum_{n=0}^{\infty} \frac{(\gamma)_{k n}}{\Gamma(\mu n+v)} \frac{t^{n}}{(\delta)_{l n}}
$$

and $(a)_{n}$ is the Pochhammer symbol: $(a)_{n}=a(a+1) \cdot \ldots \cdot(a+n-1),(a)_{0}=1$.

Remark 1. If $k=l=1$ in (3), then the integral operator $\left(\varepsilon_{\mu, \nu, 1, \omega, a^{+}}^{\gamma, \delta, k} \Psi\right)$ reduces to an integral operator $\left(\varepsilon_{\mu, v, l, \omega, a^{+}}^{\gamma, \delta} \Psi\right)$ containing generalized Mittag-Leffler function $E_{\mu, \nu, 1}^{\gamma, \delta, 1}$ introduced by Srivastava and Tomovski in [8]. Along with $k=l=1$, if $\delta=1$, then (3) reduces to an integral operator defined by Prabhaker in [9] containing Mittag-Leffler function $E_{\mu, v}^{\gamma}$. For $\omega=0$ in (3), the integral operator $\left(\varepsilon_{\mu, v, l, l, \omega, a^{+}}^{\gamma, \delta, k}\right)$ reduces to the Riemann-Liouville fractional integral operator [7]. Note that $E_{\mu, v, l}^{\gamma, \delta, k}(t)$ is absolutely convergent for all $t \in \mathbb{R}$, where $k<l+\mu$. Since $\left|E_{\mu, v, l}^{\gamma, \delta, k}(t)\right| \leq \sum_{n=0}^{\infty}\left|\frac{(\gamma)_{k n}}{\Gamma(\mu n+v)} \frac{t^{n}}{(\delta)_{l n}}\right|$ with $\sum_{n=0}^{\infty}\left|\frac{(\gamma)_{k n}}{\Gamma(\mu n+v)} \frac{t^{n}}{(\delta)_{l n}}\right|=S$, we have $\left|E_{\mu, v, l}^{\gamma, \delta, k}(t)\right| \leq S$.

Inspired by Definition 3, we give the following new definition:

Definition 4. Let $\mu, v, k, l, \gamma$ be positive real numbers and $\omega \in \mathbb{R}$, then

$$
\begin{aligned}
& \left(\varepsilon_{\mu, v, l, \omega, a^{+}, c^{+}}^{\gamma, \delta,} \Psi\right)(x, y) \\
& =\int_{a}^{x} \int_{c}^{y}(x-t)^{v_{1}-1}(y-s)^{\nu_{2}-1} E_{\mu_{1}, \nu_{1}, l_{1}}^{\gamma_{1}, \delta_{1}, k_{1}}\left(\omega_{1}(x-t)^{\mu_{1}}\right) E_{\mu_{2}, \nu_{2}, l_{2}}^{\gamma_{2}, \delta_{2}, k_{2}}\left(\omega_{2}(y-s)^{\mu_{2}}\right) \Psi(t, s) \mathrm{d} s \mathrm{~d} t
\end{aligned}
$$


$x>a, y>c$

$$
\begin{aligned}
& \left(\varepsilon_{\mu, v, l, \omega, a^{+}, d^{-}}^{\gamma, \delta} \Psi\right)(x, y) \\
& =\int_{a}^{x} \int_{y}^{d}(x-t)^{\nu_{1}-1}(s-y)^{\nu_{2}-1} E_{\mu_{1}, v_{1}, l_{1}}^{\gamma_{1}, \delta_{1}, k_{1}}\left(\omega_{1}(x-t)^{\mu_{1}}\right) E_{\mu_{2}, \nu_{2}, l_{2}}^{\gamma_{2}, \delta_{2}, k_{2}}\left(\omega_{2}(s-y)^{\mu_{2}}\right) \Psi(t, s) \mathrm{d} s \mathrm{~d} t,
\end{aligned}
$$

$x>a, y<d ;$

$$
\begin{aligned}
& \left(\varepsilon_{\mu, v, l, l, \omega, b^{-}, c^{+}}^{\gamma, \delta}\right)(x, y) \\
& =\int_{x}^{b} \int_{\mathcal{c}}^{y}(t-x)^{v_{1}-1}(y-s)^{\nu_{2}-1} E_{\mu_{1}, v_{1}, l_{1}}^{\gamma_{1}, \delta_{1}, k_{1}}\left(\omega_{1}(t-x)^{\mu_{1}}\right) E_{\mu_{2}, \nu_{2}, l_{2}}^{\gamma_{2}, \delta_{2}, k_{2}}\left(\omega_{2}(y-s)^{\mu_{2}}\right) \Psi(t, s) \mathrm{d} s \mathrm{~d} t,
\end{aligned}
$$

$x<b, y>d$ respectively

$$
\begin{aligned}
& \left(\varepsilon_{\mu, v, l, \omega, b^{-}, d^{-}}^{\gamma, \delta, k}\right)(x, y) \\
& =\int_{x}^{b} \int_{y}^{d}(t-x)^{\nu_{1}-1}(s-y)^{\nu_{2}-1} E_{\mu_{1}, \nu_{1}, l_{1}}^{\gamma_{1}, \delta_{1}, k_{1}}\left(\omega_{1}(t-x)^{\mu_{1}}\right) E_{\mu_{2}, v_{2}, l_{2}}^{\gamma_{2}, \delta_{2}, k_{2}}\left(\omega_{2}(s-y)^{\mu_{2}}\right) \Psi(t, s) \mathrm{d} s \mathrm{~d} t,
\end{aligned}
$$

$x<b, y<d$, where $\mu=\left(\mu_{1}, \mu_{2}\right), v=\left(v_{1}, v_{2}\right), \omega=\left(\omega_{1}, \omega_{2}\right), \gamma=\left(\gamma_{1}, \gamma_{2}\right), \delta=\left(\delta_{1}, \delta_{2}\right), k=$ $\left(k_{1}, k_{2}\right), \mu, v, \omega, \gamma, \delta, k>(0,0)$.

Similar to Definition 4, we introduce the following fractional integrals

Definition 5. Let $\mu, v, k, l, \gamma$ be positive real numbers and $\omega \in \mathbb{R}$, then

$$
\begin{aligned}
& \left(\varepsilon_{\mu_{1}, v_{1}, l_{1}, \omega_{1}, a^{+}}^{\gamma_{1}, \delta_{1}, k_{1}}\right) \Psi\left(x, \frac{c+d}{2}\right)=\int_{a}^{x}(x-t)^{\nu_{1}-1} E_{\mu_{1}, \nu_{1}, l_{1}}^{\gamma_{1}, \delta_{1}, k_{1}}\left(\omega_{1}(t-x)^{\mu_{1}}\right) \Psi\left(t, \frac{c+d}{2}\right) \mathrm{d} t, \\
& \left(\varepsilon_{\mu_{1}, \nu_{1}, l_{1}, \omega_{1}, b^{-}}^{\gamma_{1}, \delta_{1}, k_{1}}\right) \Psi\left(x, \frac{c+d}{2}\right)=\int_{x}^{b}(t-x)^{\nu_{1}-1} E_{\mu_{1}, \nu_{1}, l_{1}}^{\gamma_{1}, \delta_{1}, k_{1}}\left(\omega_{1}(t-x)^{\mu_{1}}\right) \Psi\left(t, \frac{c+d}{2}\right) \mathrm{d} t, \\
& \left(\varepsilon_{\mu_{2}, \nu_{2}, l_{2}, \omega_{2}, c^{+}}^{\gamma_{2}, \delta_{2}, k_{2}}\right) \Psi\left(\frac{a+b}{2}, y\right)=\int_{c}^{y}(y-s)^{\nu_{2}-1} E_{\mu_{2}, \nu_{2}, l_{2}}^{\gamma_{2}, \delta_{2}, k_{2}}\left(\omega_{2}(y-s)^{\mu_{2}}\right) \Psi\left(\frac{a+b}{2}, s\right) \mathrm{d} s, \\
& \left(\varepsilon_{\mu_{2}, \nu_{2}, l_{2}, \omega_{2}, d^{-}}^{\gamma_{2}, \delta_{2}, k_{2}}\right) \Psi\left(\frac{a+b}{2}, y\right)=\int_{y}^{d}(s-y)^{\nu_{2}-1} E_{\mu_{2}, \nu_{2}, l_{2}}^{\gamma_{2}, \delta_{2}, k_{2}}\left(\omega_{2}(s-y)^{\mu_{2}}\right) \Psi\left(\frac{a+b}{2}, s\right) \mathrm{d} s .
\end{aligned}
$$

Definition 6. A function $t \mathcal{G}: \Delta \rightarrow \mathbb{R}$ is said to be symmetric with respect to $\frac{a+b}{2}$ and $\frac{c+d}{2}$ on the coordinates if

$$
t \mathcal{G}(x, y)=\left\{\begin{array}{c}
t \mathcal{G}(a+b-x, c+d-y) \\
t \mathcal{G}(x, c+d-y) \\
t \mathcal{G}(a+b-x, y)
\end{array}\right.
$$

holds for all $x \in[a, b]$ and $y \in[c, d]$.

Lemma 1. Let $p \in \mathbb{R} \backslash\{0\}$, and $t \mathcal{G}:[a, b] \subseteq \mathbb{R} \backslash\{0\} \rightarrow \mathbb{R}$ be integrable and $p$-symmetric with respect to $\frac{a^{p}+b^{p}}{2}$, then

(i) If $p>0$,

$$
\begin{aligned}
& \left(\begin{array}{l}
\varepsilon^{\gamma, \delta, k} \\
\mu, v, l, \omega,\left(\frac{a^{p}+b^{p}}{2}\right)^{+}
\end{array} t \mathcal{G} \circ h\right)\left(b^{p}\right)=\left(\begin{array}{l}
\varepsilon^{\gamma, \delta, k} \\
\mu, v, l, \omega,\left(\frac{a^{p}+b^{p}}{2}\right)^{-}
\end{array} \mathcal{G} \circ h\right)\left(a^{p}\right) \\
& =\frac{1}{2}\left[\left(\begin{array}{l}
\varepsilon^{\gamma, \delta, k} \\
\mu, v, l, \omega,\left(\frac{a^{p}+b^{p}}{2}\right)^{+}
\end{array}+\mathcal{G} \circ h\right)\left(b^{p}\right)+\left(\begin{array}{l}
\varepsilon^{\gamma, \delta, k} \\
\mu, v, l, \omega,\left(\frac{a^{p}+b^{p}}{2}\right)^{-}
\end{array} \mathcal{G}^{\prime} \circ h\right)\left(a^{p}\right)\right],
\end{aligned}
$$


with $h(x)=x^{1 / p}, x \in\left[a^{p}, b^{p}\right]$.

(ii) If $p<0$,

$$
\begin{aligned}
& \left(\begin{array}{l}
\varepsilon^{\gamma, \delta, k} \\
\mu, v, l, \omega,\left(\frac{a^{p}+b^{p}}{2}\right)^{+}
\end{array} t \mathcal{G} \circ h\right)\left(a^{p}\right)=\left(\begin{array}{l}
\varepsilon^{\gamma, \delta, k} \\
\mu, v, l, \omega,\left(\frac{a^{p}+b^{p}}{2}\right)^{-}
\end{array}-\mathcal{G} \circ h\right)\left(b^{p}\right)
\end{aligned}
$$

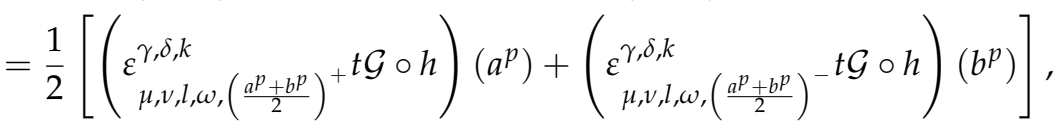

with $h(x)=x^{1 / p}, x \in\left[b^{p}, a^{p}\right]$.

For the applications of and related results containing Mittag-Leffler functions, see [10,11].

For details on Hermite-Hadamard-type inequalities involving fractional integrals via different classes of convex functions, see Kunt et al. [12], Mihai [13,14], Mihai and Mitroi [15], Nisan et al. [16], Noor et al. [17], Sarikaya and Yildirim [18], and others.

\section{Main Results}

Now we are in a position to present our main results.

Lemma 2. If the function $t \mathcal{G}: \Delta \rightarrow \mathbb{R}$ is non-negative, integrable, and symmetric with respect to $\frac{a+b}{2}$ and $\frac{c+d}{2}$ on the coordinates, then the following equalities hold:

$$
\begin{aligned}
& \left(\varepsilon_{\mu, \nu, l, \omega, b^{-}, d^{-}}^{\gamma, \delta \mathcal{G}}\right)(a, c)=\left(\varepsilon_{\mu, v, l, \omega, b^{-}, c^{+}}^{\gamma, \delta, k}\right)(a, d)=\left(\varepsilon_{\mu, v, l, \omega, a^{+}, d^{-}}^{\gamma, \delta, k}\right)(b, c)=\left(\varepsilon_{\mu, v, l, \omega, a^{+}, c^{+}}^{\gamma, \delta, k}\right)(b, d) \\
& =\frac{1}{4}\left[\left(\varepsilon_{\mu, \nu, l, \omega, b^{-}, d^{-}}^{\gamma, \delta \mathcal{G}}\right)(a, c)+\left(\varepsilon_{\mu, \nu, l, \omega, b^{-}, c^{+}}^{\gamma, \delta} t \mathcal{G}\right)(a, d)+\left(\varepsilon_{\mu, \nu, l, \omega, a^{+}, d^{-}}^{\gamma, \delta, k} t \mathcal{G}\right)(b, c)\right. \\
& \left.+\left(\varepsilon_{\mu, v, l, l, \omega, a^{+}, c^{+}}^{\gamma, \delta} \mathcal{G}\right)(b, d)\right] \text {. }
\end{aligned}
$$

Proof. Using $t \mathcal{G}$ symmetry with respect to $\frac{a+b}{2}$ and $\frac{c+d}{2}$ on the coordinates and substitutions $x=a+b-t, y=c+d-s$, we have

$$
\begin{aligned}
& \left(\varepsilon_{\mu, v, l, \omega, a^{+}, c^{+}}^{\gamma, \delta,} t \mathcal{G}\right)(b, d) \\
& =\int_{a}^{b} \int_{c}^{d}(b-t)^{v_{1}-1}(d-s)^{v_{2}-1} E_{\mu_{1}, \nu_{1}, l_{1}}^{\gamma_{1}, \delta_{1}, k_{1}}\left(\omega_{1}(b-t)^{\mu_{1}}\right) \mathcal{E}_{\mu_{2}, v_{2}, l_{2}}^{\gamma_{2}, \delta_{2}, k_{2}}\left(\omega_{2}(d-s)^{\mu_{2}}\right) t \mathcal{G}(t, s) \mathrm{d} s \mathrm{~d} t \\
& =\int_{a}^{b} \int_{c}^{d}(b-t)^{\nu_{1}-1}(d-s)^{\nu_{2}-1} \mathcal{E}_{\mu_{1}, \nu_{1}, l_{1}}^{\gamma_{1}, \delta_{1}, k_{1}}\left(\omega_{1}(b-t)^{\mu_{1}}\right) \mathcal{E}_{\mu_{2}, \nu_{2}, l_{2}}^{\gamma_{2}, \delta_{2}, k_{2}}\left(\omega_{2}(d-s)^{\mu_{2}}\right) \\
& \times t \mathcal{G}(a+b-t, c+d-s) \mathrm{d} s \mathrm{~d} t \\
& =\int_{a}^{b} \int_{c}^{d}(x-a)^{\nu_{1}-1}(y-c)^{\nu_{2}-1} \mathcal{E}_{\mu_{1}, \nu_{1}, l_{1}}^{\gamma_{1}, \delta_{1}, k_{1}}\left(\omega_{1}(x-a)^{\mu_{1}}\right) \mathcal{E}_{\mu_{2}, \nu_{2}, l_{2}}^{\gamma_{2}, \delta_{2}, k_{2}}\left(\omega_{2}(y-c)^{\mu_{2}}\right) t \mathcal{G}(x, y) \mathrm{d} y \mathrm{~d} x \\
& =\left(\varepsilon_{\mu, v, l, \omega, b^{-}, d^{-}}^{\gamma, \delta \mathcal{G}}\right)(a, c) \text {. } \\
& \left(\varepsilon_{\mu, v, l, \omega, a^{+}, d^{-}}^{\gamma, \delta, k}\right)(b, c) \\
& =\int_{a}^{b} \int_{c}^{d}(b-t)^{v_{1}-1}(s-c)^{\nu_{2}-1} \mathcal{E}_{\mu_{1}, \nu_{1}, l_{1}}^{\gamma_{1}, \delta_{1}, k_{1}}\left(\omega_{1}(b-t)^{\mu_{1}}\right) \mathcal{E}_{\mu_{2}, \nu_{2}, l_{2}}^{\gamma_{2}, \delta_{2}, k_{2}}\left(\omega_{2}(s-c)^{\mu_{2}}\right) t \mathcal{G}(t, s) \mathrm{d} s \mathrm{~d} t \\
& =\int_{a}^{b} \int_{c}^{d}(b-t)^{v_{1}-1}(s-c)^{\nu_{2}-1} \mathcal{E}_{\mu_{1}, \nu_{1}, l_{1}}^{\gamma_{1}, \delta_{1}, k_{1}}\left(\omega_{1}(b-t)^{\mu_{1}}\right) \mathcal{E}_{\mu_{2}, \nu_{2}, l_{2}}^{\gamma_{2}, \delta_{2}, k_{2}}\left(\omega_{2}(s-c)^{\mu_{2}}\right) \\
& \times t \mathcal{G}(a+b-t, c+d-s) \mathrm{d} s \mathrm{~d} t \\
& =\int_{a}^{b} \int_{c}^{d}(x-a)^{\nu_{1}-1}(d-y)^{\nu_{2}-1} \mathcal{E}_{\mu_{1}, \nu_{1}, l_{1}}^{\gamma_{1}, \delta_{1}, k_{1}}\left(\omega_{1}(x-a)^{\mu_{1}}\right) \mathcal{E}_{\mu_{2}, \nu_{2}, l_{2}}^{\gamma_{2}, \delta_{2}, k_{2}}\left(\omega_{2}(d-y)^{\mu_{2}}\right) t \mathcal{G}(x, y) \mathrm{d} y \mathrm{~d} x \\
& =\left(\varepsilon_{\mu, v, l, \omega, b^{-}, c^{+}}^{\gamma, \delta} t \mathcal{G}\right)(a, d) \text {. }
\end{aligned}
$$

Now, using $t \mathcal{G}$ with respect to $\frac{a+b}{2}$ and substitution $u=a+b-t$, we obtain 


$$
\begin{aligned}
& \left(\varepsilon_{\mu, v, l, \omega, a^{+}, c^{+}}^{\gamma, \delta, k}\right)(b, d) \\
& =\int_{a}^{b} \int_{c}^{d}(b-t)^{\nu_{1}-1}(d-s)^{\nu_{2}-1} \mathcal{E}_{\mu_{1}, \nu_{1}, l_{1}}^{\gamma_{1}, \delta_{1}, k_{1}}\left(\omega_{1}(b-t)^{\mu_{1}}\right) \mathcal{E}_{\mu_{2}, \nu_{2}, l_{2}}^{\gamma_{2}, \delta_{2}, k_{2}}\left(\omega_{2}(d-s)^{\mu_{2}}\right) t \mathcal{G}(t, s) \mathrm{d} s \mathrm{~d} t \\
& =\int_{a}^{b} \int_{c}^{d}(b-t)^{\nu_{1}-1}(d-s)^{\nu_{2}-1} \mathcal{E}_{\mu_{1}, \nu_{1}, l_{1}}^{\gamma_{1}, \gamma_{1}, k_{1}}\left(\omega_{1}(b-t)^{\mu_{1}}\right) \mathcal{E}_{\mu_{2}, \nu_{2}, l_{2}}^{\gamma_{2}, \nu_{2}, k_{2}}\left(\omega_{2}(d-s)^{\mu_{2}}\right) \\
& \times t \mathcal{G}(a+b-t, s) \mathrm{d} s \mathrm{~d} t \\
& =\int_{a}^{b} \int_{c}^{d}(u-a)^{\nu_{1}-1}(d-s)^{\nu_{2}-1} \mathcal{E}_{\mu_{1}, v_{1}, l_{1}}^{\gamma_{1}, \delta_{1}, k_{1}}\left(\omega_{1}(u-a)^{\mu_{1}}\right) \mathcal{E}_{\mu_{2}, \nu_{2}, l_{2}}^{\gamma_{2}, \delta_{2}, k_{2}}\left(\omega_{2}(d-s)^{\mu_{2}}\right) t \mathcal{G}(u, s) \mathrm{d} s \mathrm{~d} u \\
& =\left(\varepsilon_{\mu, v, l, \omega, b^{-}, c^{+}}^{\gamma, \delta} t \mathcal{G}\right)(a, d) \text {. }
\end{aligned}
$$

Combining Equations (6)-(8), we get Equation (5) and the proof is complete.

Lemma 3. Let $\Psi:[a, b] \rightarrow \mathbb{R}$ be a convex function such that $\Psi \in L[a, b]$, and let $\mu, v, l, \gamma, \delta, k>0, \omega \in \mathbb{R}$. If the function $t \mathcal{G}:[a, b] \rightarrow \mathbb{R}$ is non-negative, integrable, and symmetric with respect to $\frac{a+b}{2}$, then the following inequalities for fractional integrals hold:

$$
\begin{aligned}
& \Psi\left(\frac{a+b}{2}\right)\left[\left(\varepsilon_{\mu, v, l, \omega^{\prime}, b^{-}}^{\gamma, \delta,} \mathcal{G}\right)(a)+\left(\varepsilon_{\mu, v, l, \omega^{\prime}, a^{+}}^{\gamma, \delta} t \mathcal{G}\right)(b)\right] \leq\left[\left(\varepsilon_{\mu, v, l, l, \omega^{\prime}, b^{-}}^{\gamma, \delta, k}\right)(a)+\left(\varepsilon_{\mu, v, l, \omega^{\prime}, a^{+}}^{\gamma, \delta, k} f g\right)(b)\right] \\
& \leq \frac{\Psi(a)+\Psi(b)}{2}\left[\left(\varepsilon_{\mu, \gamma, v, l, \omega^{\prime}, b^{-}}^{\gamma, \delta} t \mathcal{G}\right)(a)+\left(\varepsilon_{\mu, v, l, l, \omega^{\prime}, a^{+}}^{\gamma, \delta}\right)(b)\right],
\end{aligned}
$$

where $\omega^{\prime}=\frac{\omega}{(b-a)^{\mu}}$.

Proof. Since $\Psi$ is a convex function on $[a, b]$, we have for all $t \in[0,1]$

$$
\Psi\left(\frac{a+b}{2}\right)=\Psi\left(\frac{t a+(1-t) b+t b+(1-t) a}{2}\right) \leq \frac{\Psi(t a+(1-t) b)+\Psi(t b+(1-t) a)}{2} .
$$

Multiplying both sides of (10) by $2 t^{\nu-1} \mathcal{E}_{\mu, v, l}^{\gamma, \delta, k}\left(\omega t^{\mu}\right) t \mathcal{G}(t b+(1-t) a)$ then integrating the resulting inequality with respect to $t$ over $[0,1]$, we obtain

$$
\begin{aligned}
& 2 \Psi\left(\frac{a+b}{2}\right) \int_{0}^{1} t^{\nu-1} \mathcal{E}_{\mu, v, l}^{\gamma, \delta, k}\left(\omega t^{\mu}\right) t \mathcal{G}(t b+(1-t) a) \mathrm{d} t \\
& \leq \int_{0}^{1} t^{\nu-1} \mathcal{E}_{\mu, v, l}^{\gamma, \delta, k}\left(\omega t^{\mu}\right) \Psi(t a+(1-t) b) t \mathcal{G}(t b+(1-t) a) \mathrm{d} t \\
& +\int_{0}^{1} t^{\nu-1} \mathcal{E}_{\mu, v, l}^{\gamma, \delta, k}\left(\omega t^{\mu}\right) \Psi(t b+(1-t) a) t \mathcal{G}(t b+(1-t) a) \mathrm{d} t .
\end{aligned}
$$

Setting $x=t b+(1-t) a$ and $\mathrm{d} x=(b-a) \mathrm{d} t$ gives

$$
\begin{aligned}
& \frac{2 \Psi\left(\frac{a+b}{2}\right)}{(b-a)^{v}} \int_{a}^{b}(x-a)^{v-1} \mathcal{E}_{\mu, v, l}^{\gamma, \delta, k}\left(\omega^{\prime}(x-a)^{\mu}\right) t \mathcal{G}(x) \mathrm{d} x \\
& \leq \frac{1}{(b-a)^{v}}\left[\int_{a}^{b}(x-a)^{\nu-1} \mathcal{E}_{\mu, v, l}^{\gamma, \delta, k}\left(\omega^{\prime}(x-a)^{\mu}\right) \Psi(a+b-x) t \mathcal{G}(x) \mathrm{d} x\right. \\
& \left.+\int_{a}^{b}(x-a)^{v-1} \mathcal{E}_{\mu, v, l}^{\gamma, \delta, k}\left(\omega^{\prime}(x-a)^{\mu}\right) \Psi(x) t \mathcal{G}(x) \mathrm{d} x\right] \\
& =\frac{1}{(b-a)^{v}}\left[\int_{a}^{b}(b-x)^{\nu-1} \mathcal{E}_{\mu, v, l}^{\gamma, \delta, k}\left(\omega^{\prime}(b-x)^{\mu}\right) \Psi(x) t \mathcal{G}(a+b-x) \mathrm{d} x\right.
\end{aligned}
$$




$$
\begin{aligned}
& \left.+\int_{a}^{b}(x-a)^{v-1} \mathcal{E}_{\mu, \delta, l, l}^{\gamma, k}\left(\omega^{\prime}(x-a)^{\mu}\right)(f g)(x) \mathrm{d} x\right] \\
& =\frac{1}{(b-a)^{v}}\left[\int_{a}^{b}(b-x)^{v-1} \mathcal{E}_{\mu, v, l}^{\gamma, \delta, k}\left(\omega^{\prime}(b-x)^{\mu}\right) \Psi(x) t \mathcal{G}(x) \mathrm{d} x\right. \\
& \left.+\int_{a}^{b}(x-a)^{v-1} \mathcal{E}_{\mu, \nu, l, l}^{\gamma, \delta}\left(\omega^{\prime}(x-a)^{\mu}\right)(f g)(x) \mathrm{d} x\right] .
\end{aligned}
$$

So

$$
2 \Psi\left(\frac{a+b}{2}\right)\left(\varepsilon_{\mu, v, l, \omega^{\prime}, b^{-}}^{\gamma, \delta, k}\right)(a) \leq\left[\left(\varepsilon_{\mu, v, l, \omega^{\prime}, b^{-}}^{\gamma, \delta g}\right)(a)+\left(\varepsilon_{\mu, v, l, \omega^{\prime}, a^{+}}^{\gamma, \delta} f g\right)(b)\right],
$$

and using Lemma 1, we have

$$
\Psi\left(\frac{a+b}{2}\right)\left[\left(\varepsilon_{\mu, v, l, \omega^{\prime}, b^{-}}^{\gamma, \delta, k}\right)(a)+\left(\varepsilon_{\mu, v, l, \omega^{\prime}, a^{+}}^{\gamma, \delta, k} t \mathcal{G}\right)(b)\right] \leq\left[\left(\varepsilon_{\mu, v, l, l, \omega^{\prime}, b^{-}}^{\gamma, \delta g}\right)(a)+\left(\varepsilon_{\mu, v, l, \omega^{\prime}, a^{+}}^{\gamma, \delta, k} f g\right)(b)\right] .
$$

The first inequality is proved.

For the proof of the second inequality of (9), we first note that if $\Psi$ is a convex function, then for all $t \in[0,1]$, it yields

$$
\Psi(t a+(1-t) b)+\Psi((1-t) a+t b) \leq \Psi(a)+\Psi(b) .
$$

Then, multiplying both sides of (11) by $t^{\nu-1} \mathcal{E}_{\mu, v, l}^{\gamma, \delta, k}\left(\omega t^{\mu}\right) t \mathcal{G}(t b+(1-t) a)$ and integrating the resulting inequality with respect to $t$ over $[0,1]$, we obtain

$$
\begin{aligned}
& \int_{0}^{1} t^{\nu-1} \mathcal{E}_{\mu, v, l}^{\gamma, \delta, k}\left(\omega t^{\mu}\right) \Psi(t a+(1-t) b) t \mathcal{G}(t b+(1-t) a) \mathrm{d} t \\
& +\int_{0}^{1} t^{\nu-1} \mathcal{E}_{\mu, v, l}^{\gamma, \delta, k}\left(\omega t^{\mu}\right) \Psi(t b+(1-t) a) t \mathcal{G}(t b+(1-t) a) \mathrm{d} t \\
& \leq(\Psi(a)+\Psi(b)) \int_{0}^{1} t^{\nu-1} \mathcal{E}_{\mu, v, l}^{\gamma, \delta, k}\left(\omega t^{\mu}\right) t \mathcal{G}(t b+(1-t) a) \mathrm{d} t .
\end{aligned}
$$

That is,

$$
\begin{aligned}
& {\left[\left(\varepsilon_{\mu, v, l, l, \omega^{\prime}, b^{-}}^{\gamma, \delta} f\right)(a)+\left(\varepsilon_{\mu, v, l, \omega^{\prime}, a^{+}}^{\gamma, \delta, k} f g\right)(b)\right]} \\
& \leq \frac{\Psi(a)+\Psi(b)}{2}\left[\left(\varepsilon_{\mu, v, l, l, \omega^{\prime}, b^{-}}^{\gamma \gamma, \delta}\right)(a)+\left(\varepsilon_{\mu, v, l, l, \omega^{\prime}, a^{+}}^{\gamma \gamma, k}\right)(b)\right] .
\end{aligned}
$$

The proof is completed.

Remark 2. If in Lemma 3 we put $\omega=(0,0)$, we obtain [19] (Theorem 2.2).

The next result is the Hermite-Hadamard-type inequality for coordinated convex functions containing the generalized Mittag-Leffler function.

Theorem 1. Let $\Psi: \Delta \rightarrow \mathbb{R}$ be coordinated convex on $\Delta=[a, b] \times[c, d]$ in $\mathbb{R}^{2}$ with $a<b, c<d$ and $\Psi \in L[\Delta]$. Then, one has the inequalities 


$$
\begin{aligned}
& \Psi\left(\frac{a+b}{2}, \frac{c+d}{2}\right)\left[\begin{array}{c}
\left(\varepsilon_{\mu, v, l, \omega^{\prime}, b^{-}, d^{-}}^{\gamma, \delta, k}\right)(a, c)+\left(\varepsilon_{\mu, v, l, \omega^{\prime}, b^{-}, c^{+}}^{\gamma, \delta}\right)(a, d) \\
+\left(\varepsilon_{\mu, v, l, \omega^{\prime}, a^{+}, d^{-}}^{\gamma, j}\right)(b, c)+\left(\varepsilon_{\mu, v, l, \omega^{\prime}, a^{+}, c^{+}}^{\gamma, \delta}\right)(b, d)
\end{array}\right] \\
& \leq\left[\begin{array}{c}
\left(\varepsilon_{\mu, v, l, \omega^{\prime}, b^{-}, d^{-}}^{\gamma, \delta} \Psi\right)(a, c)+\left(\varepsilon_{\mu, v, l, \omega^{\prime}, b^{-}, c^{+}}^{\gamma, \delta}\right)(a, d) \\
+\left(\varepsilon_{\mu, v, l, \omega^{\prime}, a^{+}, d^{-}}^{\gamma, \delta, k}\right)(b, c)+\left(\varepsilon_{\mu, v, l, \omega^{\prime}, a^{+}, c^{+}}^{\gamma, \delta, k}\right)(b, d)
\end{array}\right] \\
& \leq \frac{\Psi(a, c)+\Psi(b, c)+\Psi(a, d)+\Psi(b, d)}{4}\left[\begin{array}{c}
\left(\varepsilon_{\mu, v, l, \omega^{\prime}, b^{-}, d^{-}}^{\gamma, \delta, k}\right)(a, c)+\left(\varepsilon_{\mu, v, l, \omega^{\prime}, b^{-}, c^{+}}^{\gamma, \delta, k}\right)(a, d) \\
+\left(\varepsilon_{\mu, v, l, l, \omega^{\prime}, a^{+}, d^{-}}^{\gamma, \delta, k}\right)(b, c)+\left(\varepsilon_{\mu, v, l, \omega^{\prime}, a^{+}, c^{+}} 1\right)(b, d)
\end{array}\right],
\end{aligned}
$$

where $\mu=\left(\mu_{1}, \mu_{2}\right), v=\left(v_{1}, v_{2}\right), \omega^{\prime}=\left(\omega_{1}^{\prime}, \omega_{2}^{\prime}\right), \gamma=\left(\gamma_{1}, \gamma_{2}\right), \delta=\left(\delta_{1}, \delta_{2}\right), k=\left(k_{1}, k_{2}\right)$, $\mu, v, \omega^{\prime}, \gamma, \delta, k>(0,0)$ with $\omega_{1}^{\prime}=\frac{\omega_{1}}{(b-a)^{\mu_{1}}}, \omega_{2}^{\prime}=\frac{\omega_{2}}{(d-c)^{\mu_{2}}}$.

Proof. Using (2) with $x=t a+(1-t) b, y=(1-t) a+t b, u=s c+(1-s) d, w=(1-s) c+t d$ and $t=s=\frac{1}{2}$, we find that

$$
\begin{aligned}
& \Psi\left(\frac{a+b}{2}, \frac{c+d}{2}\right) \\
& \leq[\Psi(t a+(1-t) b, s c+(1-s) d)+\Psi(t a+(1-t) b,(1-s) c+s d) \\
& +\Psi((1-t) a+t b, s c+(1-s) d)+\Psi((1-t) a+t b,(1-s) c+s) d)] .
\end{aligned}
$$

Thus, multiplying both sides of (13) by $t^{\nu_{1}-1} s^{v_{2}-1} \mathcal{E}_{\mu_{1}, \nu_{1}, l_{1}}^{\gamma_{1}, \delta_{1}, k_{1}}\left(\omega_{1} t^{\mu_{1}}\right) \mathcal{E}_{\mu_{2}, \nu_{2}, l_{2}}^{\gamma_{2}, \delta_{2}, k_{2}}\left(\omega_{2} S^{\mu_{2}}\right)$ and by integrating with respect to $(t, s)$ on $[0,1] \times[0,1]$, we obtain

$$
\begin{aligned}
& \Psi\left(\frac{a+b}{2}, \frac{c+d}{2}\right) \int_{0}^{1} \int_{0}^{1} t^{\nu_{1}-1} s^{v_{2}-1} \mathcal{E}_{\mu_{1}, \nu_{1}, l_{1}}^{\gamma_{1}, \delta_{1}, k_{1}}\left(\omega_{1} t^{\mu_{1}}\right) \mathcal{E}_{\mu_{2}, \nu_{2}, l_{2}}^{\gamma_{2}, \delta_{2}, k_{2}}\left(\omega_{2} s^{\mu_{2}}\right) \mathrm{d} s \mathrm{~d} t
\end{aligned}
$$

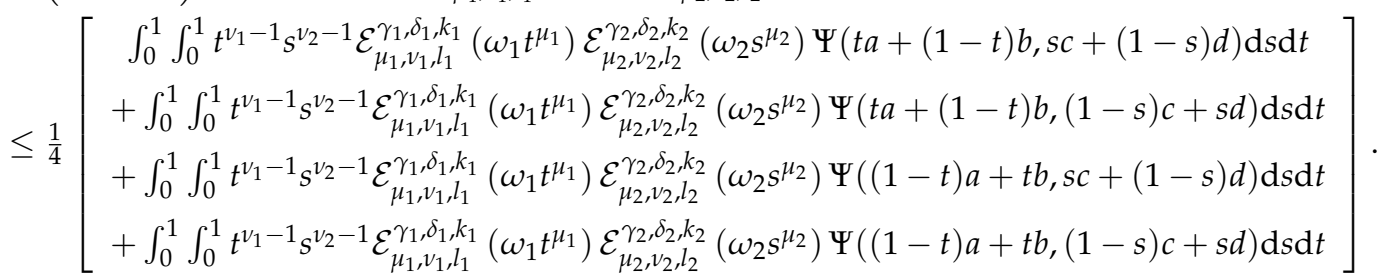

Using substitutions $u+t a+(1-t) b, v=s c+(1-s) d$, we have

$$
\begin{aligned}
& \int_{0}^{1} \int_{0}^{1} t^{v_{1}-1} s^{v_{2}-1} \mathcal{E}_{\mu_{1}, \nu_{1}, l_{1}}^{\gamma_{1}, \delta_{1}, k_{1}}\left(\omega_{1} t^{\mu_{1}}\right) \mathcal{E}_{\mu_{2}, l_{2}, l_{2}}^{\gamma_{2}, \delta_{2}, k_{2}}\left(\omega_{2} s^{\mu_{2}}\right) \mathrm{d} s \mathrm{~d} t \\
& =\frac{1}{(b-a)^{v_{1}-1}(d-c)^{v_{2}-1}} \\
& \times \int_{a}^{b} \int_{c}^{d}(b-u)^{v_{1}-1}(d-v)^{v_{2}-1} \mathcal{E}_{\mu_{1}, v_{1}, l_{1}}^{\gamma_{1}, \delta_{1}, k_{1}}\left(\omega_{1}^{\prime}(b-u)^{\mu_{1}}\right) \mathcal{E}_{\mu_{2}, v_{2}, l_{2}}^{\gamma_{2}, \delta_{2}, k_{2}}\left(\omega_{2}^{\prime}(d-v)^{\mu_{2}}\right) \mathrm{d} v \mathrm{~d} u \\
& =\frac{1}{(b-a)^{v_{1}-1}(d-c)^{v_{2}-1}}\left(\varepsilon_{\mu, v, l, \omega^{\prime}, a^{+}, c^{+}}^{\gamma, \delta, k} 1\right)(b, d),
\end{aligned}
$$

with $\omega_{1}^{\prime}=\frac{\omega_{1}}{(b-a)^{v_{1}-1}}, \omega_{2}^{\prime}=\frac{\omega_{2}}{(d-c)^{v_{2}-1}}$, and

$$
\begin{aligned}
& \int_{0}^{1} \int_{0}^{1} t^{v_{1}-1} s^{v_{2}-1} \mathcal{E}_{\mu_{1}, v_{1}, l_{1}}^{\gamma_{1}, \delta_{1}, k_{1}}\left(\omega_{1} t^{\mu_{1}}\right) \mathcal{E}_{\mu_{2}, v_{2}, l_{2}}^{\gamma_{2}, \delta_{2}, k_{2}}\left(\omega_{2} s^{\mu_{2}}\right) \Psi(t a+(1-t) b, s c+(1-s) d) \mathrm{d} s \mathrm{~d} t \\
& =\frac{1}{(b-a)^{v_{1}-1}(d-c)^{v_{2}-1}} \\
& \times \int_{a}^{b} \int_{c}^{d}(b-u)^{v_{1}-1}(d-v)^{v_{2}-1} \mathcal{E}_{\mu_{1}, \nu_{1}, l_{1}}^{\gamma_{1}, \delta_{1}, k_{1}}\left(\omega_{1}^{\prime}(b-u)^{\mu_{1}}\right) \mathcal{E}_{\mu_{2}, v_{2}, l_{2}}^{\gamma_{2}, \delta_{2}, k_{2}}\left(\omega_{2}^{\prime}(d-v)^{\mu_{2}}\right) \Psi(u, v) \mathrm{d} v \mathrm{~d} u \\
& =\frac{1}{(b-a)^{v_{1}-1}(d-c)^{v_{2}-1}}\left(\varepsilon_{\mu, v, l, \omega^{\prime}, a^{+}, c^{+}}^{\gamma, \delta, k}\right)(b, d) .
\end{aligned}
$$


Analogously, we obtain

$$
\begin{aligned}
& \int_{0}^{1} \int_{0}^{1} t^{\nu_{1}-1} s^{\nu_{2}-1} \mathcal{E}_{\mu_{1}, \nu_{1}, l_{1}}^{\gamma_{1}, \delta_{1}, k_{1}}\left(\omega_{1} t^{\mu_{1}}\right) \mathcal{E}_{\mu_{2}, \nu_{2}, l_{2}}^{\gamma_{2}, \delta_{2}, k_{2}}\left(\omega_{2} s^{\mu_{2}}\right) \Psi(t a+(1-t) b,(1-s) c+s d) \mathrm{d} s \mathrm{~d} t \\
& =\frac{1}{(b-a)^{v_{1}-1}(d-c)^{v_{2}-1}}\left(\varepsilon_{\mu, \nu, l, \omega^{\prime}, a^{+}, d^{-}}^{\gamma, \delta} \Psi\right)(b, c), \\
& \int_{0}^{1} \int_{0}^{1} t^{\nu_{1}-1} s^{v_{2}-1} \mathcal{E}_{\mu_{1}, \nu_{1}, l_{1}}^{\gamma_{1}, \delta_{1}, k_{1}}\left(\omega_{1} t^{\mu_{1}}\right) \mathcal{E}_{\mu_{2}, \nu_{2}, l_{2}}^{\gamma_{2}, \delta_{2}, k_{2}}\left(\omega_{2} s^{\mu_{2}}\right) \Psi((1-t) a+t b, s c+(1-s) d) \mathrm{d} s \mathrm{~d} t \\
& =\frac{1}{(b-a)^{v_{1}-1}(d-c)^{v_{2}-1}}\left(\varepsilon_{\mu, v, l, \omega^{\prime}, b^{-}, c^{+}}^{\gamma, \delta, k} \Psi\right)(a, d), \\
& \int_{0}^{1} \int_{0}^{1} t^{\nu_{1}-1} s^{\nu_{2}-1} \mathcal{E}_{\mu_{1}, \nu_{1}, l_{1}}^{\gamma_{1}, \delta_{1}, k_{1}}\left(\omega_{1} t^{\mu_{1}}\right) \mathcal{E}_{\mu_{2}, \nu_{2}, l_{2}}^{\gamma_{2}, \delta_{2}, k_{2}}\left(\omega_{2} s^{\mu_{2}}\right) \Psi((1-t) a+t b,(1-s) c+s d) \mathrm{d} s \mathrm{~d} t \\
& =\frac{1}{(b-a)^{v_{1}-1}(d-c)^{v_{2}-1}}\left(\varepsilon_{\mu, \nu, l, \omega^{\prime}, b^{-}, d^{-}}^{\gamma, \delta, k} \Psi\right)(a, c) \text {. }
\end{aligned}
$$

Using Lemma 2, introducing relationships (15)-(19) in (14) and after multiplying with $(b-a)^{v_{1}-1}(d-c)^{v_{2}-1}$, we get

$$
\begin{aligned}
& \Psi\left(\frac{a+b}{2}, \frac{c+d}{2}\right)\left[\begin{array}{c}
\left(\varepsilon_{\mu, v, l, \omega^{\prime}, b^{-}, d^{-}}^{\gamma, \delta, k}\right)(a, c)+\left(\varepsilon_{\mu, v, l, \omega^{\prime}, b^{-}, c^{+}}^{\gamma, \delta}\right)(a, d) \\
+\left(\varepsilon_{\mu, v, l, \omega^{\prime}, a^{+}, d^{-}}^{\gamma, \delta, k}\right)(b, c)+\left(\varepsilon_{\mu, v, l, \omega^{\prime}, a^{+}, c^{+}}^{\gamma, \delta, k}\right)(b, d)
\end{array}\right] \\
& \leq\left[\begin{array}{c}
\left(\varepsilon_{\mu, v, l, \omega^{\prime}, b^{-}, d^{-}}^{\gamma, \delta, k}\right)(a, c)+\left(\varepsilon_{\mu, v, l, \omega^{\prime}, b^{-}, c^{+}}^{\gamma, \delta}\right)(a, d) \\
+\left(\varepsilon_{\mu, v, l, l, \omega^{\prime}, a^{+}, d^{-}}^{\gamma, \delta}\right)(b, c)+\left(\varepsilon_{\mu, v, l, \omega^{\prime}, a^{+}, c^{+}}^{\gamma, \delta, k}\right)(b, d)
\end{array}\right],
\end{aligned}
$$

by which the first inequality of (12) is proved.

For the proof of the second inequality in (12) using (2), we have

$$
\begin{aligned}
& \Psi(t a+(1-t) b, s c+(1-s) d)+\Psi(t a+(1-t) b,(1-s) c+s d) \\
& +\Psi((1-t) a+t b, s c+(1-s) d)+\Psi((1-t) a+t b,(1-s) c+s d) \\
& \leq \Psi(a, c)+\Psi(b, c)+\Psi(a, d)+\Psi(b, d) .
\end{aligned}
$$

Then, multiplying both sides of (21) by $t^{\nu_{1}-1} s^{\nu_{2}-1} \mathcal{E}_{\mu_{1}, \nu_{1}, l_{1}}^{\gamma_{1}, \delta_{1}, k_{1}}\left(\omega_{1} t^{\mu_{1}}\right) \mathcal{E}_{\mu_{2}, \nu_{2}, l_{2}}^{\gamma_{2}, \delta_{2}, k_{2}}\left(\omega_{2} s^{\mu_{2}}\right)$ and integrating with respect to $(t, s)$ on $[0,1] \times[0,1]$, we get

$$
\begin{aligned}
& \int_{0}^{1} \int_{0}^{1} t^{\nu_{1}-1} s^{\nu_{2}-1} \mathcal{E}_{\mu_{1}, v_{1}, l_{1}}^{\gamma_{1}, \delta_{1}, k_{1}}\left(\omega_{1} t^{\mu_{1}}\right) \mathcal{E}_{\mu_{2}, v_{2}, l_{2}}^{\gamma_{2}, \delta_{2}, k_{2}}\left(\omega_{2} s^{\mu_{2}}\right) \Psi(t a+(1-t) b, s c+(1-s) d) \mathrm{d} s \mathrm{~d} t \\
& +\int_{0}^{1} \int_{0}^{1} t^{\nu_{1}-1} s^{\nu_{2}-1} \mathcal{E}_{\mu_{1}, \nu_{1}, l_{1}}^{\gamma_{1}, \delta_{1}, k_{1}}\left(\omega_{1} t^{\mu_{1}}\right) \mathcal{E}_{\mu_{2}, \nu_{2}, l_{2}}^{\gamma_{2}, \delta_{2}, k_{2}}\left(\omega_{2} s^{\mu_{2}}\right) \Psi(t a+(1-t) b,(1-s) c+s d) \mathrm{d} s \mathrm{~d} t \\
& +\int_{0}^{1} \int_{0}^{1} t^{\nu_{1}-1} s^{\nu_{2}-1} \mathcal{E}_{\mu_{1}, v_{1}, l_{1}}^{\gamma_{1}, \delta_{1}, k_{1}}\left(\omega_{1} t^{\mu_{1}}\right) \mathcal{E}_{\mu_{2}, \nu_{2}, l_{2}}^{\gamma_{2}, \delta_{2}, k_{2}}\left(\omega_{2} s^{\mu_{2}}\right) \Psi((1-t) a+t b, s c+(1-s) d) \mathrm{d} s \mathrm{~d} t \\
& +\int_{0}^{1} \int_{0}^{1} t^{\nu_{1}-1} s^{\nu_{2}-1} \mathcal{E}_{\mu_{1}, v_{1}, l_{1}}^{\gamma_{1}, \delta_{1}, k_{1}}\left(\omega_{1} t^{\mu_{1}}\right) \mathcal{E}_{\mu_{2}, \nu_{2}, l_{2}}^{\gamma_{2}, \delta_{2}, k_{2}}\left(\omega_{2} s^{\mu_{2}}\right) \Psi((1-t) a+t b,(1-s) c+s d) \mathrm{d} s \mathrm{~d} t \\
& \leq \int_{0}^{1} \int_{0}^{1} t^{\nu_{1}-1} s^{v_{2}-1} \mathcal{E}_{\mu_{1}, v_{1}, l_{1}}^{\gamma_{1}, \delta_{1}, k_{1}}\left(\omega_{1} t^{\mu_{1}}\right) \mathcal{E}_{\mu_{2}, v_{2}, l_{2}}^{\gamma_{2}, \delta_{2}, k_{2}}\left(\omega_{2} s^{\mu_{2}}\right)\left[\begin{array}{c}
\Psi(a, c)+\Psi(b, c) \\
+\Psi(a, d)+\Psi(b, d)
\end{array}\right] \mathrm{d} s \mathrm{~d} t .
\end{aligned}
$$

So, after multiplying with $(b-a)^{v_{1}-1}(d-c)^{v_{2}-1}$ and using Lemma 2 , we have

$$
\begin{aligned}
& {\left[\begin{array}{c}
\left(\varepsilon_{\mu, v, l, \omega^{\prime}, b^{-}, d^{-}}^{\gamma, \delta, k}\right)(a, c)+\left(\varepsilon_{\mu, v, l, \omega^{\prime}, b^{-}, c^{+}}^{\gamma, \delta} \Psi\right)(a, d) \\
+\left(\varepsilon_{\mu, v, l, l, \omega^{\prime}, a^{+}, d^{-}}^{\gamma, k}\right)(b, c)+\left(\varepsilon_{\mu, v, l, \omega^{\prime}, a^{+}, c^{+}}^{\gamma, \delta}\right)(b, d)
\end{array}\right]}
\end{aligned}
$$

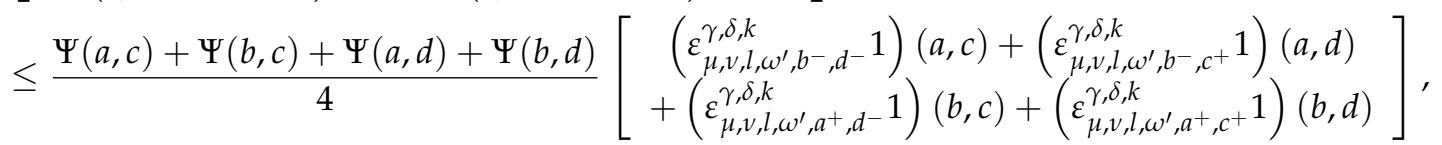


which finishes the proof.

The following theorem establishes Hermite-Hadamard-Fejér-type inequalities for coordinated convex functions containing the generalized Mittag-Leffler function.

Theorem 2. Let $\Psi: \Delta \rightarrow \mathbb{R}$ be coordinated convex on $\Delta=[a, b] \times[c, d]$ in $\mathbb{R}^{2}$ with $a<b, c<d$ and $\Psi \in L[\Delta]$. If $t \mathcal{G}: \Delta \rightarrow \mathbb{R}$ is non-negative, integrable, and symmetric with respect to $\frac{a+b}{2}, \frac{c+d}{2}$ on the coordinates, then the following integral inequalities hold:

$$
\begin{aligned}
& \Psi\left(\frac{a+b}{2}, \frac{c+d}{2}\right)\left[\begin{array}{c}
\left(\varepsilon_{\mu, \gamma, l, \omega^{\prime}, b^{-}, d^{-}}^{\gamma, \delta \mathcal{G}}\right)(a, c)+\left(\varepsilon_{\mu, \nu, l, l, \omega^{\prime}, b^{-}, c^{+}}^{\gamma, \delta,} t \mathcal{G}\right)(a, d) \\
+\left(\varepsilon_{\mu, v, l, \omega^{\prime}, a^{+}, d^{-}}^{\gamma, \delta}\right)(b, c)+\left(\varepsilon_{\mu, v, l, \omega^{\prime}, a^{+}, c^{+}}^{\gamma, \mathcal{G}}\right)(b, d)
\end{array}\right] \\
& \leq\left[\begin{array}{c}
\left(\varepsilon_{\mu, v, l, l, \omega^{\prime}, b^{-}, d^{-}}^{\gamma} f g\right)(a, c)+\left(\varepsilon_{\mu, \delta, l, l, \omega^{\prime}, b^{-}, c^{+}}^{\gamma} f g\right)(a, d) \\
+\left(\varepsilon_{\mu, v, l, l, \omega^{\prime}, a^{+}, d^{-}}^{\gamma, \delta g}\right)(b, c)+\left(\varepsilon_{\mu, v, l, l, \omega^{\prime}, a^{+}, c^{+}}^{\gamma, \delta g}\right)(b, d)
\end{array}\right] \\
& \leq \frac{\Psi(a, c)+\Psi(b, c)+\Psi(a, d)+\Psi(b, d)}{4}\left[\begin{array}{c}
\left(\varepsilon_{\mu, \nu, l, l, \omega^{\prime}, b^{-}, d^{-}}^{\gamma} t \mathcal{G}\right)(a, c)+\left(\varepsilon_{\mu, \nu, l, l, \omega^{\prime}, b^{-}, c^{+}}^{\gamma} t \mathcal{G}\right)(a, d) \\
+\left(\varepsilon_{\mu, v, l, l, \omega^{\prime}, a^{+}, d^{-}}^{\gamma, \mathcal{G}}\right)(b, c)+\left(\varepsilon_{\mu, v, l, \omega^{\prime}, a^{+}, c^{+}}^{\gamma, \mathcal{G}}\right)(b, d)
\end{array}\right],
\end{aligned}
$$

where $\mu=\left(\mu_{1}, \mu_{2}\right), v=\left(v_{1}, v_{2}\right), \omega^{\prime}=\left(\omega_{1}^{\prime}, \omega_{2}^{\prime}\right), \gamma=\left(\gamma_{1}, \gamma_{2}\right), \delta=\left(\delta_{1}, \delta_{2}\right), k=\left(k_{1}, k_{2}\right)$, $\mu, v, \omega^{\prime}, \gamma, \delta, k>(0,0)$ with $\omega_{1}^{\prime}=\frac{\omega_{1}}{(b-a)^{\mu_{1}}}, \omega_{2}^{\prime}=\frac{\omega_{2}}{(d-c)^{\mu_{2}}}$.

Proof. Since $\Psi$ is a convex function on $\Delta$, then for all $t, s \in[0,1] \times[0,1]$, we can write

$$
\begin{aligned}
& \Psi\left(\frac{a+b}{2}, \frac{c+d}{2}\right)=\Psi\left(\frac{t a+(1-t) b+(1-t) a+t b}{2}, \frac{s c+(1-s) d+(1-s) c+s d}{2}\right) \\
& \leq \frac{1}{4}\left[\begin{array}{c}
\Psi(t a+(1-t) b, s c+(1-s) d)+\Psi(t a+(1-t) b,(1-s) c+s d) \\
+\Psi((1-t) a+t b, s c+(1-s) d)+\Psi((1-t) a+t b,(1-s) c+s d)
\end{array}\right] .
\end{aligned}
$$

Then, multiplying both sides of (23) by $t^{\nu_{1}-1} \mathcal{s}^{\nu_{2}-1} \mathcal{E}_{\mu_{1}, \nu_{1}, l_{1}}^{\gamma_{1}, \delta_{1}, k_{1}}\left(\omega_{1} t^{\mu_{1}}\right) \mathcal{E}_{\mu_{2}, \nu_{2}, l_{2}}^{\gamma_{2}, \delta_{2}, k_{2}}\left(\omega_{2} s^{\mu_{2}}\right) t \mathcal{G}((1-t) a+t b$, $(1-s) c+s d)$ and integrating with respect to $(t, s)$ on $[0,1] \times[0,1]$, we obtain

$$
\begin{aligned}
& \Psi\left(\frac{a+b}{2}, \frac{c+d}{2}\right) \\
& \times \int_{0}^{1} \int_{0}^{1} t^{\nu_{1}-1} s^{v_{2}-1} \mathcal{E}_{\mu_{1}, \nu_{1}, l_{1}}^{\gamma_{1}, \delta_{1}, k_{1}}\left(\omega_{1} t^{\mu_{1}}\right) \mathcal{E}_{\mu_{2}, \nu_{2}, l_{2}}^{\gamma_{2}, \delta_{2}, k_{2}}\left(\omega_{2} s^{\mu_{2}}\right) t \mathcal{G}((1-t) a+t b,(1-s) c+s d) \mathrm{d} s \mathrm{~d} t
\end{aligned}
$$

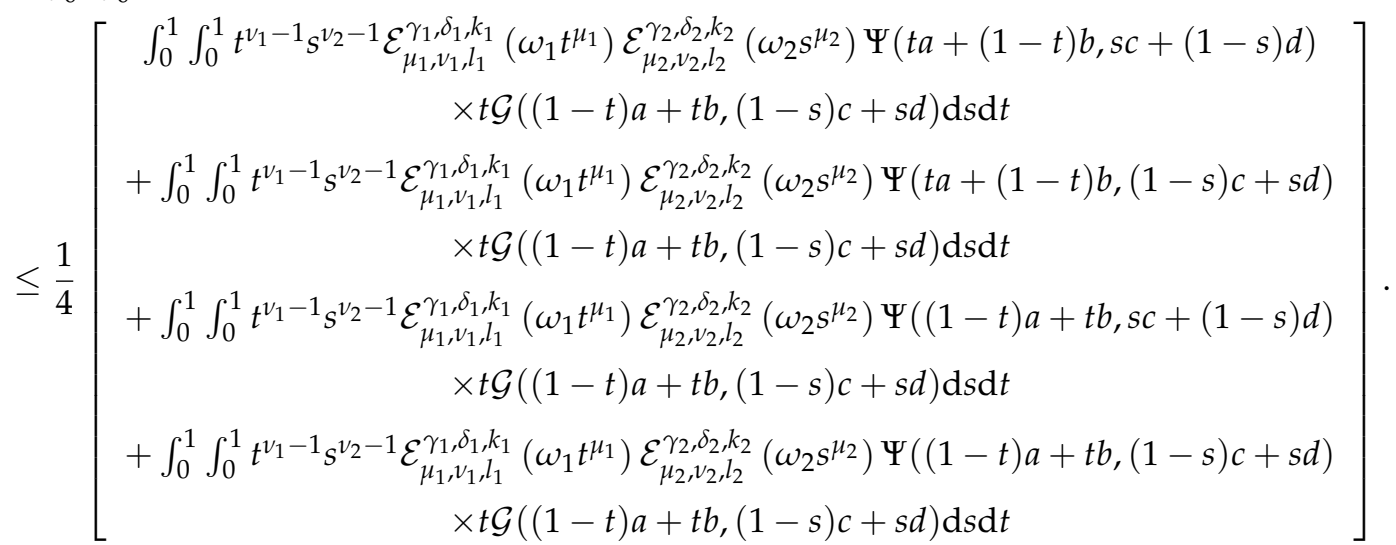

Setting $x=(1-t) a+t b$ and $y=(1-s) c+s d$, we obtain

$$
\begin{aligned}
& I_{1}=\int_{0}^{1} \int_{0}^{1} t^{\nu_{1}-1} s^{\nu_{2}-1} \mathcal{E}_{\mu_{1}, v_{1}, l_{1}}^{\gamma_{1}, \delta_{1}, k_{1}}\left(\omega_{1} t^{\mu_{1}}\right) \mathcal{E}_{\mu_{2}, v_{2}, l_{2}}^{\gamma_{2}, \delta_{2}, k_{2}}\left(\omega_{2} s^{\mu_{2}}\right) t \mathcal{G}((1-t) a+t b,(1-s) c+s d) \mathrm{d} s \mathrm{~d} t \\
& =\frac{1}{(b-a)^{\nu_{1}-1}(d-c)^{\nu_{2}-1}}\left(\mathcal{E}_{\mu, v, l, l, \omega^{\prime}, b^{-}, d^{-}}^{\gamma, \mathcal{G}}\right)(a, c)
\end{aligned}
$$


and

$$
\begin{aligned}
& I_{2}=\int_{0}^{1} \int_{0}^{1} t^{\nu_{1}-1} s^{\nu_{2}-1} \mathcal{E}_{\mu_{1}, \nu_{1}, l_{1}}^{\gamma_{1}, \delta_{1}, k_{1}}\left(\omega_{1} t^{\mu_{1}}\right) \mathcal{E}_{\mu_{2}, \nu_{2}, l_{2}}^{\gamma_{2}, \delta_{2}, k_{2}}\left(\omega_{2} s^{\mu_{2}}\right) \Psi(t a+(1-t) b, s c+(1-s) d) \\
& t \mathcal{G}((1-t) a+t b,(1-s) c+s d) \mathrm{d} s \mathrm{~d} t \\
& =\frac{1}{(b-a)^{\nu_{1}-1}(d-c)^{\nu_{2}-1}} \\
& \times \int_{a}^{b} \int_{c}^{d}(x-a)^{\nu_{1}-1}(y-c)^{\nu_{2}-1} \mathcal{E}_{\mu_{1}, \nu_{1}, l_{1}}^{\gamma_{1}, \delta_{1}, k_{1}}\left(\omega_{1}^{\prime}(x-a)^{\mu_{1}}\right) \mathcal{E}_{\mu_{2}, \nu_{2}, l_{2}}^{\gamma_{2}, \delta_{2}, k_{2}}\left(\omega_{2}^{\prime}(y-c)^{\mu_{2}}\right) \\
& \times \Psi(a+b-x, c+d-y) t \mathcal{G}(x, y) \mathrm{d} y \mathrm{~d} x .
\end{aligned}
$$

Now, using substitutions $u=a+b-x, v=c+d-y$ and the symmetry of function $t \mathcal{G}$, we have

$$
\begin{aligned}
& I_{2}=\frac{1}{(b-a)^{v_{1}-1}(d-c)^{v_{2}-1}} \\
& \times \int_{a}^{b} \int_{c}^{d}(b-u)^{v_{1}-1}(d-v)^{v_{2}-1} \mathcal{E}_{\mu_{1}, \nu_{1}, l_{1}}^{\gamma_{1}, \delta_{1}, k_{1}}\left(\omega_{1}^{\prime}(b-u)^{\mu_{1}}\right) \mathcal{E}_{\mu_{2}, v_{2}, l_{2}}^{\gamma_{2}, \delta_{2}, k_{2}}\left(\omega_{2}^{\prime}(d-v)^{\mu_{2}}\right) \\
& \times \Psi(u, v) t \mathcal{G}(a+b-u, c+d-v) \mathrm{d} v \mathrm{~d} u \\
& =\frac{1}{(b-a)^{v_{1}-1}(d-c)^{v_{2}-1}} \\
& \times \int_{a}^{b} \int_{\mathcal{c}}^{d}(b-u)^{\nu_{1}-1}(d-v)^{\nu_{2}-1} \mathcal{E}_{\mu_{1}, \nu_{1}, l_{1}}^{\gamma_{1}, \delta_{1}, k_{1}}\left(\omega_{1}^{\prime}(b-u)^{\mu_{1}}\right) \mathcal{E}_{\mu_{2}, v_{2}, l_{2}}^{\gamma_{2}, \delta_{2}, k_{2}}\left(\omega_{2}^{\prime}(d-v)^{\mu_{2}}\right) \\
& \times \Psi(u, v) t \mathcal{G}(u, v) \mathrm{d} v \mathrm{~d} u \\
& =\frac{1}{(b-a)^{v_{1}-1}(d-c)^{\nu_{2}-1}}\left(\varepsilon_{\mu, v, l, l, \omega^{\prime}, a^{+}, c^{+}}^{\gamma, \delta,} f g\right)(b, d) \text {. }
\end{aligned}
$$

Analogously, we get

$$
\begin{aligned}
& I_{3}=\int_{0}^{1} \int_{0}^{1} t^{\nu_{1}-1} s^{\nu_{2}-1} \mathcal{E}_{\mu_{1}, \nu_{1}, l_{1}}^{\gamma_{1}, \delta_{1}, k_{1}}\left(\omega_{1} t^{\mu_{1}}\right) \mathcal{E}_{\mu_{2}, \nu_{2}, l_{2}}^{\gamma_{2}, \delta_{2}, k_{2}}\left(\omega_{2} s^{\mu_{2}}\right) \Psi(t a+(1-t) b,(1-s) c+s d) \\
& \times t \mathcal{G}((1-t) a+t b,(1-s) c+s d) \mathrm{d} s \mathrm{~d} t \\
& =\frac{1}{(b-a)^{v_{1}-1}(d-c)^{v_{2}-1}} \\
& \times \int_{a}^{b} \int_{c}^{d}(x-a)^{\nu_{1}-1}(y-c)^{\nu_{2}-1} \mathcal{E}_{\mu_{1}, \nu_{1}, l_{1}}^{\gamma_{1}, \delta_{1}, k_{1}}\left(\omega_{1}^{\prime}(x-a)^{\mu_{1}}\right) \mathcal{E}_{\mu_{2}, \nu_{2}, l_{2}}^{\gamma_{2}, \delta_{2}, k_{2}}\left(\omega_{2}^{\prime}(y-c)^{\mu_{2}}\right) \\
& \times \Psi(a+b-x, y) t \mathcal{G}(x, y) \mathrm{d} y \mathrm{~d} x \\
& =\frac{1}{(b-a)^{v_{1}-1}(d-c)^{v_{2}-1}} \\
& \times \int_{a}^{b} \int_{c}^{d}(b-u)^{\nu_{1}-1}(y-c)^{\nu_{2}-1} \mathcal{E}_{\mu_{1}, l_{1}, l_{1}}^{\gamma_{1}, \delta_{1}, k_{1}}\left(\omega_{1}^{\prime}(b-u)^{\mu_{1}}\right) \mathcal{E}_{\mu_{2}, \nu_{2}, l_{2}}^{\gamma_{2}, \delta_{2}, k_{2}}\left(\omega_{2}^{\prime}(y-c)^{\mu_{2}}\right) \\
& \times \Psi(u, y) t \mathcal{G}(a+b-u, y) \mathrm{d} y \mathrm{~d} u \\
& =\frac{1}{(b-a)^{v_{1}-1}(d-c)^{v_{2}-1}} \\
& \times \int_{a}^{b} \int_{c}^{d}(b-u)^{\nu_{1}-1}(y-c)^{\nu_{2}-1} \mathcal{E}_{\mu_{1}, \nu_{1}, l_{1}}^{\gamma_{1}, \delta_{1}, k_{1}}\left(\omega_{1}^{\prime}(b-u)^{\mu_{1}}\right) \mathcal{E}_{\mu_{2}, v_{2}, l_{2}}^{\gamma_{2}, \delta_{2}, k_{2}}\left(\omega_{2}^{\prime}(y-c)^{\mu_{2}}\right) \\
& \times \Psi(u, y) t \mathcal{G}(u, y) \mathrm{d} y \mathrm{~d} u \\
& =\frac{1}{(b-a)^{v_{1}-1}(d-c)^{\nu_{2}-1}}\left(\varepsilon_{\mu, v, l, \omega^{\prime}, a^{+}, d^{-}}^{\gamma, \delta g}\right)(b, c),
\end{aligned}
$$




$$
\begin{aligned}
& I_{4}=\int_{0}^{1} \int_{0}^{1} t^{v_{1}-1} s^{v_{2}-1} \mathcal{E}_{\mu_{1}, v_{1}, l_{1}}^{\gamma_{1}, \delta_{1}, k_{1}}\left(\omega_{1} t^{\mu_{1}}\right) \mathcal{E}_{\mu_{2}, \nu_{2}, l_{2}}^{\gamma_{2}, \delta_{2}, k_{2}}\left(\omega_{2} s^{\mu_{2}}\right) \Psi((1-t) a+t b, s c+(1-s) d) \\
& \times t \mathcal{G}((1-t) a+t b,(1-s) c+s d) \mathrm{d} s \mathrm{~d} t \\
& =\frac{1}{(b-a)^{v_{1}-1}(d-c)^{v_{2}-1}} \\
& \times \int_{a}^{b} \int_{c}^{d}(x-a)^{v_{1}-1}(y-c)^{v_{2}-1} \mathcal{E}_{\mu_{1}, v_{1}, l_{1}}^{\gamma_{1}, \delta_{1}, k_{1}}\left(\omega_{1}^{\prime}(x-a)^{\mu_{1}}\right) \mathcal{E}_{\mu_{2}, v_{2}, l_{2}}^{\gamma_{2}, \delta_{2}, k_{2}}\left(\omega_{2}^{\prime}(y-c)^{\mu_{2}}\right) \\
& \times \Psi(x, c+d-y) t \mathcal{G}(x, y) \mathrm{d} y \mathrm{~d} x \\
& =\frac{1}{(b-a)^{v_{1}-1}(d-c)^{v_{2}-1}} \\
& \times \int_{a}^{b} \int_{c}^{d}(x-a)^{v_{1}-1}(d-v)^{v_{2}-1} \mathcal{E}_{\mu_{1}, v_{1}, l_{1}}^{\gamma_{1}, \delta_{1}, k_{1}}\left(\omega_{1}^{\prime}(x-a)^{\mu_{1}}\right) \mathcal{E}_{\mu_{2}, v_{2}, l_{2}}^{\gamma_{2}, \delta_{2}, k_{2}}\left(\omega_{2}^{\prime}(d-v)^{\mu_{2}}\right) \\
& \times \Psi(x, v) t \mathcal{G}(x, c+d-v) \mathrm{d} v \mathrm{~d} x \\
& =\frac{1}{(b-a)^{v_{1}-1}(d-c)^{v_{2}-1}} \\
& \times \int_{a}^{b} \int_{c}^{d}(x-a)^{v_{1}-1}(d-v)^{v_{2}-1} \mathcal{E}_{\mu_{1}, v_{1}, l_{1}}^{\gamma_{1}, \delta_{1}, k_{1}}\left(\omega_{1}^{\prime}(x-a)^{\mu_{1}}\right) \mathcal{E}_{\mu_{2}, v_{2}, l_{2}}^{\gamma_{2}, \delta_{2}, k_{2}}\left(\omega_{2}^{\prime}(d-v)^{\mu_{2}}\right) \\
& \times \Psi(x, v) t \mathcal{G}(x, v) \mathrm{d} v \mathrm{~d} x \\
& =\frac{1}{(b-a)^{v_{1}-1}(d-c)^{v_{2}-1}}\left(\varepsilon_{\mu, v, l, \omega^{\prime}, b^{-}, c^{+}}^{\gamma, \delta g}\right)(a, d),
\end{aligned}
$$

and

$$
\begin{aligned}
& I_{5}=\int_{0}^{1} \int_{0}^{1} t^{\nu_{1}-1} s^{v_{2}-1} \mathcal{E}_{\mu_{1}, \nu_{1}, l_{1}}^{\gamma_{1}, \delta_{1}, k_{1}}\left(\omega_{1} t^{\mu_{1}}\right) \mathcal{E}_{\mu_{2}, v_{2}, l_{2}}^{\gamma_{2}, \delta_{2}, k_{2}}\left(\omega_{2} s^{\mu_{2}}\right) \Psi((1-t) a+t b,(1-s) c+s d) \\
& \times t \mathcal{G}((1-t) a+t b,(1-s) c+s d) \mathrm{d} s \mathrm{~d} t \\
& =\frac{1}{(b-a)^{v_{1}-1}(d-c)^{v_{2}-1}} \\
& \times \int_{a}^{b} \int_{c}^{d}(x-a)^{v_{1}-1}(y-c)^{v_{2}-1} \mathcal{E}_{\mu_{1}, v_{1}, l_{1}}^{\gamma_{1}, \delta_{1}, k_{1}}\left(\omega_{1}^{\prime}(x-a)^{\mu_{1}}\right) \mathcal{E}_{\mu_{2}, v_{2}, l_{2}}^{\gamma_{2}, \delta_{2}, k_{2}}\left(\omega_{2}^{\prime}(y-c)^{\mu_{2}}\right) \\
& \times \Psi(x, y) t \mathcal{G}(x, y) \mathrm{d} y \mathrm{~d} x \\
& =\frac{1}{(b-a)^{v_{1}-1}(d-c)^{v_{2}-1}}\left(\varepsilon_{\mu, v, l, l, \omega^{\prime}, b^{-}, d^{-}}^{\gamma g}\right)(a, c) .
\end{aligned}
$$

Introducing (25)-(29) in (24), multiplying the inequality with $(b-a)^{v_{1}-1}(d-c)^{v_{2}-1}$, and using Lemma 2, we have the first inequality of (22).

We shall prove the second inequality of (22). Since $\Psi$ is a convex function on $\Delta$, for all $(t, s) \in$ $[0,1] \times[0,1]$, it yields

$$
\begin{aligned}
& \Psi(t a+(1-t) b, s c+(1-s) d)+\Psi(t a+(1-t) b,(1-s) c+s d) \\
& +\Psi((1-t) a+t b, s c+(1-s) d)+\Psi((1-t) a+t b,(1-s) c+s d) \\
& \leq \Psi(a, c)+\Psi(b, c)+\Psi(a, d)+\Psi(b, d) .
\end{aligned}
$$

Multiplying both sides of (30) by $t^{\nu_{1}-1} s^{\nu_{2}-1} \mathcal{E}_{\mu_{1}, v_{1}, l_{1}}^{\gamma_{1}, \delta_{1}, k_{1}}\left(\omega_{1} t^{\mu_{1}}\right) \mathcal{E}_{\mu_{2}, v_{2}, l_{2}}^{\gamma_{2}, \delta_{2}, k_{2}}\left(\omega_{2} S^{\mu_{2}}\right) t \mathcal{G}((1-t) a+t b,(1-$ $s) c+s d)$ and integrating with respect to $(t, s)$ on $[0,1] \times[0,1]$, we obtain

$$
I_{2}+I_{3}+I_{4}+I_{5} \leq(\Psi(a, c)+\Psi(b, c)+\Psi(a, d)+\Psi(b, d)) I_{1} .
$$

That is, using (25)-(29),

$$
\begin{aligned}
& \frac{1}{4}\left[\begin{array}{c}
\left(\varepsilon_{\mu, v, l, \omega^{\prime}, b^{-}, d^{-}}^{\gamma, \delta, k}\right)(a, c)+\left(\varepsilon_{\mu, v, l, \omega^{\prime}, b^{-}, c^{+}}^{\gamma, \delta} f g\right)(a, d) \\
+\left(\varepsilon_{\mu, v, l, \omega^{\prime}, a^{+}, d^{-}}^{\gamma g}\right)(b, c)+\left(\varepsilon_{\mu, v, l, \omega^{\prime}, a^{+}, c^{+}}^{\gamma, \delta} f g\right)(b, d)
\end{array}\right] \\
& \leq \frac{\Psi(a, c)+\Psi(b, c)+\Psi(a, d)+\Psi(b, d)}{4}\left(\varepsilon_{\mu, v, l, \omega^{\prime}, b^{-}, d^{-}}^{\gamma, \delta}+\mathcal{G}\right)(a, c) .
\end{aligned}
$$


Finally, by using Lemma 2 we get the second part of inequality (22), and the proof is complete.

Remark 3. If we take $\omega=(0,0)$ in Theorem 2, we have [20] (Theorem 7).

Theorem 3. Let $\Psi: \Delta \rightarrow \mathbb{R}$ be coordinated convex on $\Delta=[a, b] \times[c, d]$ in $\mathbb{R}^{2}$ with $a<b, c<d$ and $\Psi \in L[\Delta]$. If $t \mathcal{G}: \Delta \rightarrow \mathbb{R}$ is non-negative, integrable, and symmetric with respect to $\frac{a+b}{2}, \frac{c+d}{2}$ on the coordinates, then the following integral inequalities hold:

$$
\begin{aligned}
& \Psi\left(\frac{a+b}{2}, \frac{c+d}{2}\right)\left[\begin{array}{c}
\left(\varepsilon_{\mu, v, l, \omega^{\prime}, b^{-}, d^{-}}^{\gamma, \delta, k}\right)(a, c)+\left(\varepsilon_{\mu, v, l, \omega^{\prime}, b^{-}, c^{+}}^{\gamma, \delta} t \mathcal{G}\right)(a, d) \\
+\left(\varepsilon_{\mu, v, l, \omega^{\prime}, a^{+}, d^{-}}^{\gamma, \delta, k}\right)(b, c)+\left(\varepsilon_{\mu, v, l, \omega^{\prime}, a^{+}, c^{+}}^{\gamma, \delta, k}\right)(b, d)
\end{array}\right]
\end{aligned}
$$

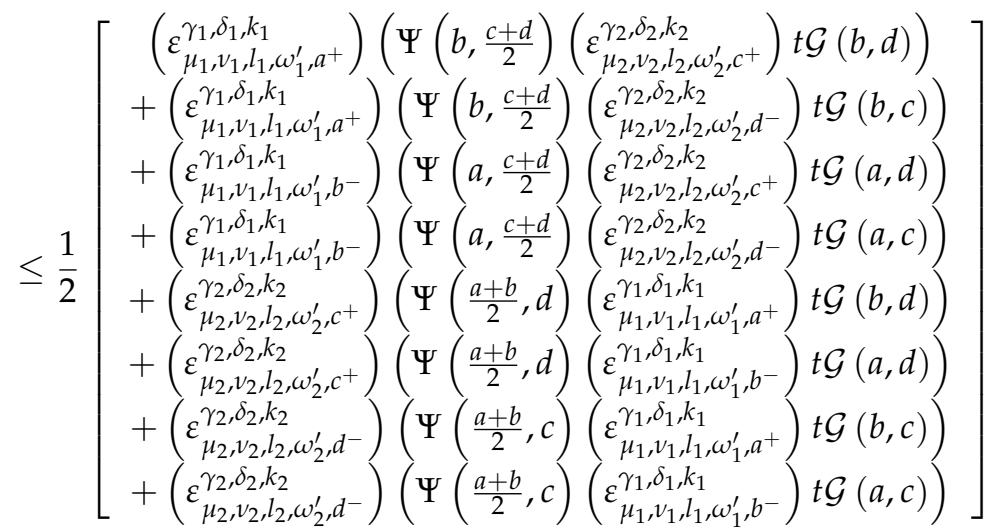

$$
\begin{aligned}
& \leq\left[\begin{array}{c}
\left(\varepsilon_{\mu, v, l, \omega^{\prime}, b^{-}, d^{-}}^{\gamma, \delta g}\right)(a, c)+\left(\varepsilon_{\mu, v, l, \omega^{\prime}, b^{-}, c^{+}}^{\gamma, \delta g}\right)(a, d) \\
+\left(\varepsilon_{\mu, v, l, \omega^{\prime}, a^{+}, d^{-}}^{\gamma, \delta g}\right)(b, c)+\left(\varepsilon_{\mu, v, l, l, \omega^{\prime}, a^{+}, c^{+}}^{\gamma, \delta g}\right)(b, d)
\end{array}\right] \\
& {\left[\begin{array}{rl} 
& \left(\varepsilon_{\mu_{1}, v_{1}, l_{1}, \omega_{1}^{\prime}, a^{+}}^{\gamma_{1}, \delta_{1}, k_{1}}\right)\left(\Psi(b, c)\left(\varepsilon_{\mu_{2}, \nu_{2}, l_{2}, \omega_{2}^{\prime}, c^{+}}^{\gamma_{2}, \delta_{2}, k_{2}}\right) t \mathcal{G}(b, d)\right) \\
+ & \left(\varepsilon^{\gamma_{1}, \delta_{1}, k_{1}}\right)\left(\Psi(b, d)\left(\varepsilon_{\gamma_{2}, \delta_{2}, k_{2}}\right) t \mathcal{G}(b, c)\right)
\end{array}\right.} \\
& +\left(\varepsilon_{\mu_{1}, v_{1}, l_{1}, \omega_{1}^{\prime}, a^{+}}^{\gamma_{1}, \delta_{1}, k_{1}}\right)\left(\Psi(b, d)\left(\varepsilon_{\mu_{2}, v_{2}, l_{2}, \omega_{2}^{\prime}, d^{-}}^{\gamma_{2}, \delta_{2}, k_{2}}\right) t \mathcal{G}(b, c)\right) \\
& +\left(\varepsilon_{\mu_{1}, v_{1}, l_{1}, \omega_{1}^{\prime}, b^{-}}^{\gamma_{1}, \delta_{1}, k_{1}}\right)\left(\Psi(a, c)\left(\varepsilon_{\mu_{2}, \nu_{2}, l_{2}, \omega_{2}^{\prime}, c^{+}}^{\gamma_{2}, \delta_{2}, k_{2}}\right) t \mathcal{G}(a, d)\right)
\end{aligned}
$$

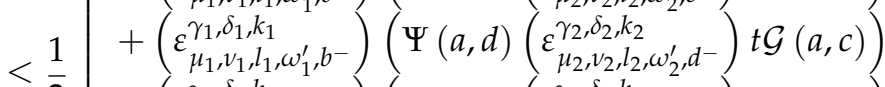

$$
\begin{aligned}
& +\left(\varepsilon_{\mu_{2}, \nu_{2}, l_{2}, \omega_{2}^{\prime}, c^{+}}^{\gamma_{2}, \delta_{2}, k_{2}}\right)\left(\Psi(a, d)\left(\varepsilon_{\mu_{1}, \nu_{1}, l_{1}, \omega_{1}^{\prime}, a^{+}}^{\gamma_{1}, \delta_{1}, k_{1}}\right) t \mathcal{G}(b, d)\right) \\
& +\left(\varepsilon_{\mu_{2}, \nu_{2}, l_{2}, \omega_{2}^{\prime}, c^{+}}^{\gamma_{2}, \delta_{2}, k_{2}}\right)\left(\Psi(b, d)\left(\varepsilon_{\varepsilon_{1}, \nu_{1}, l_{1}, \omega_{1}^{\prime}, b^{-}}^{\gamma_{1}, \delta_{1}, k_{1}}\right) t \mathcal{G}(a, d)\right) \\
& +\left(\varepsilon_{\mu_{2}, v_{2}, l_{2}, \omega_{2}^{\prime}, d^{-}}^{\gamma_{2}, \delta_{2}, k_{2}}\right)\left(\Psi(a, c)\left(\varepsilon_{\mu_{1}, v_{1}, l_{1}, \omega_{1}^{\prime}, a^{+}}^{\gamma_{1}, \delta_{1}, k_{1}}\right) t \mathcal{G}(b, c)\right) \\
& +\left(\begin{array}{c}
\varepsilon_{2} \gamma_{2}, \delta_{2}, k_{2} \\
\mu_{2}, l_{2}, l_{2}, \omega_{2}^{\prime}, d^{-}
\end{array}\right)\left(\Psi(b, c)\left(\begin{array}{c}
\varepsilon^{\gamma_{1}, \delta_{1}, k_{1}} \\
\left.\left.\mu_{\mu_{1}, \nu_{1}, l_{1}, \omega_{1}^{\prime}, b^{-}}^{\gamma_{2}}\right) t \mathcal{G}(a, c)\right)
\end{array}\right)\right.
\end{aligned}
$$

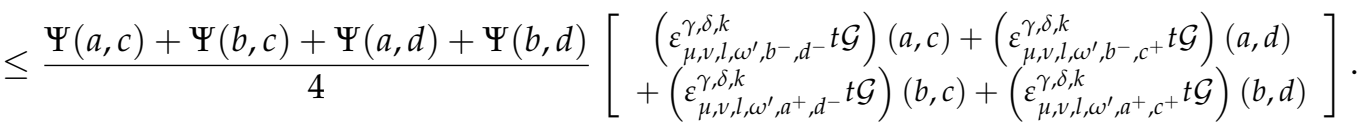

Proof. For an easier proof, we will use the following notations:

$\varepsilon_{\mu_{1}, v_{1}, l_{1}, \omega_{1}^{\prime}, a^{+}}^{\gamma_{1}, \delta_{1}, k_{1}}=\varepsilon_{a^{+}}, \varepsilon_{\mu_{1}, v_{1}, l_{1}, \omega_{1}^{\prime}, b^{-}}^{\gamma_{1}, \delta_{1}, k_{1}}=\varepsilon_{b^{-}}, \varepsilon_{\mu_{2}, v_{2}, l_{2}, \omega_{2}^{\prime}, c^{+}}^{\gamma_{2}, \delta_{2}, k_{2}}=\varepsilon_{c^{+}}, \varepsilon_{\mu_{2}, \nu_{2}, l_{2}, \omega_{2}^{\prime}, d^{-}}^{\gamma_{2}, \delta_{2}, k_{2}}=\varepsilon_{d^{-}}$, $\mathcal{E}_{\mu_{1}, \nu_{1}, l_{1}}^{\gamma_{1}, \delta_{1}, k_{1}}=\mathcal{E}_{1}, \mathcal{E}_{\mu_{2}, \nu_{2}, l_{2}}^{\gamma_{2}, \delta_{2}, k_{2}}=\mathcal{E}_{2}, \varepsilon_{\mu, v, l, \omega^{\prime}, b^{-}, d^{-}}^{\gamma, \delta, k}=\varepsilon_{b^{-}, d^{-}}, \varepsilon_{\mu, v, l, \omega^{\prime}, b^{-}, c^{+}}^{\gamma, \delta, k}=\varepsilon_{b^{-}, c^{+},} \varepsilon_{\mu, v, l, \omega^{\prime}, a^{+}, d^{-}}^{\gamma, \delta, k}=\varepsilon_{a^{+}, d^{-}}$, and $\varepsilon_{\mu, v, l, \omega^{\prime}, a^{+}, c^{+}}^{\gamma, \delta, k}=\varepsilon_{a^{+}, c^{+}}$. 
Since $\Psi: \Delta \rightarrow \mathbb{R}$ is convex on the coordinates, it follows that the mapping $\Psi_{x}:[c, d] \rightarrow$ $\mathbb{R}, \Psi_{x}(y)=\Psi(x, y)$ is convex on $[c, d]$ and $t \mathcal{G}_{x}:[c, d] \rightarrow \mathbb{R}, t \mathcal{G}_{x}=t \mathcal{G}(x, y)$ is non-negative, integrable, and symmetric with respect to $\frac{c+d}{2}$, for all $x \in[a, b]$. Then, thanks to inequalities (9) we have

$$
\begin{aligned}
& \Psi_{x}\left(\frac{c+d}{2}\right)\left[\left(\varepsilon_{c^{+}} t \mathcal{G}_{x}\right)(d)+\left(\varepsilon_{d^{-}} t \mathcal{G}_{x}\right)(c)\right] \leq\left[\left(\varepsilon_{c^{+}} \Psi_{x} t \mathcal{G}_{x}\right)(d)+\left(\varepsilon_{d^{-}} \Psi_{x} t \mathcal{G}_{x}\right)(c)\right] \\
& \leq \frac{\Psi_{x}(c)+\Psi_{x}(d)}{2}\left[\left(\varepsilon_{c^{+}} t \mathcal{G}_{x}\right)(d)+\left(\varepsilon_{d^{-}} t \mathcal{G}_{x}\right)(c)\right] .
\end{aligned}
$$

That is,

$$
\begin{aligned}
& \Psi\left(x, \frac{c+d}{2}\right)\left[\begin{array}{c}
\int_{c}^{d}(d-y)^{v_{2}-1} \mathcal{E}_{2}\left(\omega_{2}^{\prime}(d-y)^{\mu_{2}}\right) t \mathcal{G}(x, y) \mathrm{d} y \\
+\int_{c}^{d}(y-c)^{v_{2}-1} \mathcal{E}_{2}\left(\omega_{2}^{\prime}(y-c)^{\mu_{2}}\right) t \mathcal{G}(x, y) \mathrm{d} y
\end{array}\right] \\
& \leq\left[\begin{array}{c}
\int_{c}^{d}(d-y)^{v_{2}-1} \mathcal{E}_{2}\left(\omega_{2}^{\prime}(d-y)^{\mu_{2}}\right) \Psi(x, y) t \mathcal{G}(x, y) \mathrm{d} y \\
+\int_{c}^{d}(y-c)^{v_{2}-1} \mathcal{E}_{2}\left(\omega_{2}^{\prime}(y-c)^{\mu_{2}}\right) \Psi(x, y) t \mathcal{G}(x, y) \mathrm{d} y
\end{array}\right] \\
& \leq \frac{\Psi(x, c)+\Psi(x, d)}{2}\left[\begin{array}{c}
\int_{c}^{d}(d-y)^{v_{2}-1} \mathcal{E}_{2}\left(\omega_{2}^{\prime}(d-y)^{\mu_{2}}\right) t \mathcal{G}(x, y) \mathrm{d} y \\
+\int_{c}^{d}(y-c)^{\nu_{2}-1} \mathcal{E}_{2}\left(\omega_{2}^{\prime}(y-c)^{\mu_{2}}\right) t \mathcal{G}(x, y) \mathrm{d} y
\end{array}\right] .
\end{aligned}
$$

Multiplying both sides of (32) by $(b-x)^{\nu_{1}-1} \mathcal{E}_{1}\left(\omega_{1}^{\prime}(b-x)^{\mu_{1}}\right)$ and $(x-a)^{\nu_{1}-1} \mathcal{E}_{1}\left(\omega_{1}^{\prime}(x-a)^{\mu_{1}}\right)$, and integrating with respect to $x$ over $[a, b]$, respectively, we have

$$
\begin{aligned}
& \int_{a}^{b} \int_{c}^{d}(b-x)^{v_{1}-1}(d-y)^{\nu_{2}-1} \mathcal{E}_{1}\left(\omega_{1}^{\prime}(b-x)^{\mu_{1}}\right) \mathcal{E}_{2}\left(\omega_{2}^{\prime}(d-y)^{\mu_{2}}\right) \Psi\left(x, \frac{c+d}{2}\right) t \mathcal{G}(x, y) \mathrm{d} y \mathrm{~d} x \\
& +\int_{a}^{b} \int_{c}^{d}(b-x)^{v_{1}-1}(y-c)^{v_{2}-1} \mathcal{E}_{1}\left(\omega_{1}^{\prime}(b-x)^{\mu_{1}}\right) E_{2}\left(\omega_{2}^{\prime}(y-c)^{\mu_{2}}\right) \Psi\left(x, \frac{c+d}{2}\right) t \mathcal{G}(x, y) \mathrm{d} y \mathrm{~d} x \\
& \leq \int_{a}^{b} \int_{c}^{d}(b-x)^{v_{1}-1}(d-y)^{v_{2}-1} \mathcal{E}_{1}\left(\omega_{1}^{\prime}(b-x)^{\mu_{1}}\right) \mathcal{E}_{2}\left(\omega_{2}^{\prime}(d-y)^{\mu_{2}}\right) \Psi(x, y) t \mathcal{G}(x, y) \mathrm{d} y \mathrm{~d} x \\
& +\int_{a}^{b} \int_{c}^{d}(b-x)^{v_{1}-1}(y-c)^{v_{2}-1} \mathcal{E}_{1}\left(\omega_{1}^{\prime}(b-x)^{\mu_{1}}\right) \mathcal{E}_{2}\left(\omega_{2}^{\prime}(y-c)^{\mu_{2}}\right) \Psi(x, y) t \mathcal{G}(x, y) \mathrm{d} y \mathrm{~d} x \\
& \leq \frac{1}{2}\left[\begin{array}{c}
\int_{a}^{b} \int_{c}^{d}(b-x)^{\nu_{1}-1}(d-y)^{v_{2}-1} \mathcal{E}_{1}\left(\omega_{1}^{\prime}(b-x)^{\mu_{1}}\right) \mathcal{E}_{2}\left(\omega_{2}^{\prime}(d-y)^{\mu_{2}}\right) \Psi(x, c) t \mathcal{G}(x, y) \mathrm{d} y \mathrm{~d} x \\
+\int_{a}^{b} \int_{c}^{d}(b-x)^{v_{1}-1}(y-c)^{\nu_{2}-1} \mathcal{E}_{1}\left(\omega_{1}^{\prime}(b-x)^{\mu_{1}}\right) \mathcal{E}_{2}\left(\omega_{2}^{\prime}(y-c)^{\mu_{2}}\right) \Psi(x, d) t \mathcal{G}(x, y) \mathrm{d} y \mathrm{~d} x
\end{array}\right], \\
& \int_{a}^{b} \int_{c}^{d}(x-a)^{\nu_{1}-1}(d-y)^{\nu_{2}-1} \mathcal{E}_{1}\left(\omega_{1}^{\prime}(x-a)^{\mu_{1}}\right) \mathcal{E}_{2}\left(\omega_{2}^{\prime}(d-y)^{\mu_{2}}\right) \Psi\left(x, \frac{c+d}{2}\right) t \mathcal{G}(x, y) \mathrm{d} y \mathrm{~d} x \\
& +\int_{a}^{b} \int_{c}^{d}(x-a)^{v_{1}-1}(y-c)^{\nu_{2}-1} \mathcal{E}_{1}\left(\omega_{1}^{\prime}(x-a)^{\mu_{1}}\right) \mathcal{E}_{2}\left(\omega_{2}^{\prime}(y-c)^{\mu_{2}}\right) \Psi\left(x, \frac{c+d}{2}\right) t \mathcal{G}(x, y) \mathrm{d} y \mathrm{~d} x \\
& \leq \int_{a}^{b} \int_{c}^{d}(x-a)^{v_{1}-1}(d-y)^{\nu_{2}-1} \mathcal{E}_{1}\left(\omega_{1}^{\prime}(x-a)^{\mu_{1}}\right) \mathcal{E}_{2}\left(\omega_{2}^{\prime}(d-y)^{\mu_{2}}\right) \Psi(x, y) t \mathcal{G}(x, y) \mathrm{d} y \mathrm{~d} x \\
& +\int_{a}^{b} \int_{c}^{d}(x-a)^{\nu_{1}-1}(y-c)^{\nu_{2}-1} \mathcal{E}_{1}\left(\omega_{1}^{\prime}(x-a)^{\mu_{1}}\right) \mathcal{E}_{2}\left(\omega_{2}^{\prime}(y-c)^{\mu_{2}}\right) \Psi(x, y) t \mathcal{G}(x, y) \mathrm{d} y \mathrm{~d} x \\
& \leq \frac{1}{2}\left[\begin{array}{c}
\int_{a}^{b} \int_{c}^{d}(x-a)^{\nu_{1}-1}(d-y)^{\nu_{2}-1} \mathcal{E}_{1}\left(\omega_{1}^{\prime}(x-a)^{\mu_{1}}\right) \mathcal{E}_{2}\left(\omega_{2}^{\prime}(d-y)^{\mu_{2}}\right) \Psi(x, c) t \mathcal{G}(x, y) \mathrm{d} y \mathrm{~d} x \\
+\int_{a}^{b} \int_{c}^{d}(x-a)^{\nu_{1}-1}(y-c)^{\nu_{2}-1} \mathcal{E}_{1}\left(\omega_{1}^{\prime}(x-a)^{\mu_{1}}\right) \mathcal{E}_{2}\left(\omega_{2}^{\prime}(y-c)^{\mu_{2}}\right) \Psi(x, d) t \mathcal{G}(x, y) \mathrm{d} y \mathrm{~d} x
\end{array}\right] .
\end{aligned}
$$

For the mappings $\Psi_{y}:[a, b] \rightarrow \mathbb{R}, \Psi_{y}=\Psi(x, y)$ and $t \mathcal{G}_{y}:[a, b] \rightarrow \mathbb{R}, t \mathcal{G}_{y}=t \mathcal{G}(x, y)$, we use the same arguments as before. So we can state that 


$$
\begin{aligned}
& \int_{a}^{b} \int_{c}^{d}(b-x)^{\nu_{1}-1}(d-y)^{\nu_{2}-1} \mathcal{E}_{1}\left(\omega_{1}^{\prime}(b-x)^{\mu_{1}}\right) \mathcal{E}_{2}\left(\omega_{2}^{\prime}(d-y)^{\mu_{2}}\right) \Psi\left(\frac{a+b}{2}, y\right) t \mathcal{G}(x, y) \mathrm{d} y \mathrm{~d} x \\
& +\int_{a}^{b} \int_{c}^{d}(x-a)^{\nu_{1}-1}(d-y)^{\nu_{2}-1} \mathcal{E}_{1}\left(\omega_{1}^{\prime}(x-a)^{\mu_{1}}\right) \mathcal{E}_{2}\left(\omega_{2}^{\prime}(d-y)^{\mu_{2}}\right) \Psi\left(\frac{a+b}{2}, y\right) t \mathcal{G}(x, y) \mathrm{d} y \mathrm{~d} x \\
& \leq \frac{1}{2}\left[\begin{array}{c}
\int_{a}^{b} \int_{c}^{d}(b-x)^{\nu_{1}-1}(d-y)^{\nu_{2}-1} \mathcal{E}_{1}\left(\omega_{1}^{\prime}(b-x)^{\mu_{1}}\right) \mathcal{E}_{2}\left(\omega_{2}^{\prime}(d-y)^{\mu_{2}}\right) \Psi(a, y) t \mathcal{G}(x, y) \mathrm{d} y \mathrm{~d} x \\
+\int_{a}^{b} \int_{c}^{d}(x-a)^{\nu_{1}-1}(d-y)^{\nu_{2}-1} \mathcal{E}_{1}\left(\omega_{1}^{\prime}(x-a)^{\mu_{1}}\right) \mathcal{E}_{2}\left(\omega_{2}^{\prime}(d-y)^{\mu_{2}}\right) \Psi(b, y) t \mathcal{G}(x, y) \mathrm{d} y \mathrm{~d} x
\end{array}\right]
\end{aligned}
$$

and

$$
\begin{aligned}
& \int_{a}^{b} \int_{c}^{d}(b-x)^{v_{1}-1}(y-c)^{v_{2}-1} \mathcal{E}_{1}\left(\omega_{1}^{\prime}(b-x)^{\mu_{1}}\right) \mathcal{E}_{2}\left(\omega_{2}^{\prime}(y-c)^{\mu_{2}}\right) \Psi\left(\frac{a+b}{2}, y\right) t \mathcal{G}(x, y) \mathrm{d} y \mathrm{~d} x \\
& +\int_{a}^{b} \int_{c}^{d}(x-a)^{\nu_{1}-1}(y-c)^{\nu_{2}-1} \mathcal{E}_{1}\left(\omega_{1}^{\prime}(x-a)^{\mu_{1}}\right) \mathcal{E}_{2}\left(\omega_{2}^{\prime}(y-c)^{\mu_{2}}\right) \Psi\left(\frac{a+b}{2}, y\right) t \mathcal{G}(x, y) \mathrm{d} y \mathrm{~d} x \\
& \leq \frac{1}{2}\left[\begin{array}{c}
\int_{a}^{b} \int_{c}^{d}(b-x)^{\nu_{1}-1}(y-c)^{\nu_{2}-1} \mathcal{E}_{1}\left(\omega_{1}^{\prime}(b-x)^{\mu_{1}}\right) \mathcal{E}_{2}\left(\omega_{2}^{\prime}(y-c)^{\mu_{2}}\right) \Psi(a, y) t \mathcal{G}(x, y) \mathrm{d} y \mathrm{~d} x \\
+\int_{a}^{b} \int_{c}^{d}(x-a)^{v_{1}-1}(y-c)^{\nu_{2}-1} \mathcal{E}_{1}\left(\omega_{1}^{\prime}(x-a)^{\mu_{1}}\right) \mathcal{E}_{2}\left(\omega_{2}^{\prime}(y-c)^{\mu_{2}}\right) \Psi(b, y) t \mathcal{G}(x, y) \mathrm{d} y \mathrm{~d} x
\end{array}\right]
\end{aligned}
$$

Adding the inequalities (33)-(36), we can write

$$
\begin{aligned}
& \varepsilon_{a^{+}}\left[\Psi\left(b, \frac{c+d}{2}\right) \varepsilon_{c^{+}} t \mathcal{G}(b, d)\right]+\varepsilon_{a^{+}}\left[\Psi\left(b, \frac{c+d}{2}\right) \varepsilon_{d^{-}} t \mathcal{G}(b, c)\right] \\
& +\varepsilon_{b^{-}}\left[\Psi\left(a, \frac{c+d}{2}\right) \varepsilon_{c^{+}} t \mathcal{G}(a, d)\right]+\varepsilon_{b^{-}}\left[\Psi\left(a, \frac{c+d}{2}\right) \varepsilon_{d^{-}} t \mathcal{G}(a, c)\right] \\
& +\varepsilon_{c^{+}}\left[\Psi\left(\frac{a+b}{2}, d\right) \varepsilon_{a^{+}} t \mathcal{G}(b, d)\right]+\varepsilon_{c^{+}}\left[\Psi\left(\frac{a+b}{2}, d\right) \varepsilon_{b^{-}} t \mathcal{G}(a, d)\right] \\
& +\varepsilon_{d^{-}}\left[\Psi\left(\frac{a+b}{2}, d\right) \varepsilon_{a^{+}} t \mathcal{G}(b, c)\right]+\varepsilon_{d^{-}}\left[\Psi\left(\frac{a+b}{2}, c\right) \varepsilon_{b^{-}} t \mathcal{G}(a, c)\right] \\
& \leq 2\left[\left(\varepsilon_{a^{+} c^{+}} f g\right)(b, d)+\left(\varepsilon_{a^{+} d^{-}} f g\right)(b, c)+\left(\varepsilon_{b^{-}} c^{+} f g\right)(a, d)+\left(\varepsilon_{b^{-} d^{-}} f g\right)(a, c)\right] \\
& \leq \frac{1}{2}\left[\begin{array}{c}
\varepsilon_{a^{+}}\left[\Psi(b, c) \varepsilon_{c^{+}} t \mathcal{G}(b, d)\right]+\varepsilon_{a^{+}}\left[\Psi(b, d) \varepsilon_{d^{-}} t \mathcal{G}(b, c)\right] \\
+\varepsilon_{c^{+}}\left[\Psi(a, c) \varepsilon_{c^{+}} t \mathcal{G}(a, d)\right]+\varepsilon_{b^{-}}\left[\Psi(a, d) \varepsilon_{a^{+}} t \mathcal{G}(b, d)\right]+\varepsilon_{c^{+}}\left[\Psi(b, d) \varepsilon_{b^{-}} t \mathcal{G}(a, d)\right] \\
+\varepsilon_{d^{-}}\left[\Psi(a, c) \varepsilon_{a^{+}} t \mathcal{G}(b, c)\right]+\varepsilon_{d^{-}}\left[\Psi(b, c) \varepsilon_{b^{-}} t \mathcal{G}(a, c)\right]
\end{array}\right] .
\end{aligned}
$$

So,

$$
\begin{aligned}
& \frac{1}{2}\left\{\begin{array}{c}
\varepsilon_{a^{+}}\left[\Psi\left(b, \frac{c+d}{2}\right) \varepsilon_{c^{+}} t \mathcal{G}(b, d)\right]+\varepsilon_{a^{+}}\left[\Psi\left(b, \frac{c+d}{2}\right) \varepsilon_{d^{-}} t \mathcal{G}(b, c)\right] \\
+\varepsilon_{b^{-}}\left[\Psi\left(a, \frac{c+d}{2}\right) \varepsilon_{c^{+}} t \mathcal{G}(a, d)\right]+\varepsilon_{b^{-}}\left[\Psi\left(a, \frac{c+d}{2}\right) \varepsilon_{d^{-}} t \mathcal{G}(a, c)\right] \\
+\varepsilon_{c^{+}}\left[\Psi\left(\frac{a+b}{2}, d\right) \varepsilon_{a^{+}} t \mathcal{G}(b, d)\right]+\varepsilon_{c^{+}}\left[\Psi\left(\frac{a+b}{2}, d\right) \varepsilon_{b^{-}} t \mathcal{G}(a, d)\right] \\
+\varepsilon_{d^{-}}\left[\Psi\left(\frac{a+b}{2}, d\right) \varepsilon_{a^{+}} t \mathcal{G}(b, c)\right]+\varepsilon_{d^{-}}\left[\Psi\left(\frac{a+b}{2}, c\right) \varepsilon_{b^{-}} t \mathcal{G}(a, c)\right]
\end{array}\right\} \\
& \leq\left[\left(\varepsilon_{a^{+} c^{+}} f g\right)(b, d)+\left(\varepsilon_{a^{+} d^{-}} f g\right)(b, c)+\left(\varepsilon_{b^{-} c^{+}} f g\right)(a, d)+\left(\varepsilon_{b^{-} d^{-}} f g\right)(a, c)\right] \\
& \leq \frac{1}{4}\left[\begin{array}{c}
\varepsilon_{a^{+}}\left[\Psi(b, c) \varepsilon_{c^{+}} t \mathcal{G}(b, d)\right]+\varepsilon_{a^{+}}\left[\Psi(b, d) \varepsilon_{d^{-}} t \mathcal{G}(b, c)\right] \\
+\varepsilon_{b^{-}}\left[\Psi(a, c) \varepsilon_{c^{+}} t \mathcal{G}(a, d)\right]+\varepsilon_{b^{-}}\left[\Psi(a, d) \varepsilon_{d^{-}} t \mathcal{G}(a, c)\right] \\
+\varepsilon_{c^{+}}\left[\Psi(a, d) \varepsilon_{a^{+}} t \mathcal{G}(b, d)\right]+\varepsilon_{c^{+}}\left[\Psi(b, d) \varepsilon_{b^{-}} t \mathcal{G}(a, d)\right] \\
+\varepsilon_{d^{-}}\left[\Psi(a, c) \varepsilon_{a^{+}} t \mathcal{G}(b, c)\right]+\varepsilon_{d^{-}}\left[\Psi(b, c) \varepsilon_{b^{-}} t \mathcal{G}(a, c)\right]
\end{array}\right] \\
& \leq \frac{1}{2}\left[\begin{array}{c}
\varepsilon_{a^{+}}\left[\Psi(b, c) \varepsilon_{c^{+}} t \mathcal{G}(b, d)\right]+\varepsilon_{a^{+}}\left[\Psi(b, d) \varepsilon_{d^{-}} t \mathcal{G}(b, c)\right] \\
+\varepsilon_{b^{-}}\left[\Psi(a, c) \varepsilon_{c^{+}} t \mathcal{G}(a, d)\right]+\varepsilon_{b^{-}}\left[\Psi(a, d) \varepsilon_{d^{-}} \mathcal{H}(a, c)\right] \\
+\varepsilon_{c^{+}}\left[\Psi(a, d) \varepsilon_{a^{+}} t \mathcal{G}(b, d)\right]+\varepsilon_{c^{+}}\left[\Psi(b, d) \varepsilon_{b^{-}} t \mathcal{G}(a, d)\right] \\
+\varepsilon_{d^{-}}\left[\Psi(a, c) \varepsilon_{a^{+}} t \mathcal{G}(b, c)\right]+\varepsilon_{d^{-}}\left[\Psi(b, c) \varepsilon_{b^{-}} t \mathcal{G}(a, c)\right]
\end{array}\right] .
\end{aligned}
$$


This gives the second and third inequalities in (31).

Now we will show the first inequality in (31).

Apply Lemma 3 for the functions $F_{1}:[a, b] \rightarrow \mathbb{R}, F_{1}(x)=\Psi\left(x, \frac{c+d}{2}\right)$ and $t \mathcal{G}_{y}:[a, b] \rightarrow$ $\mathbb{R}, t \mathcal{G}_{y}(x)=t \mathcal{G}(x, y)$ :

$$
F_{1}\left(\frac{a+b}{2}\right)\left[\left(\varepsilon_{b^{-}}+\mathcal{G}_{y}\right)(a)+\left(\varepsilon_{a^{+}} t \mathcal{G}_{y}\right)(b)\right] \leq\left[\left(\varepsilon_{b^{-}} F_{1} t \mathcal{G}_{y}\right)(a)+\left(\varepsilon_{a^{+}} F_{1} t \mathcal{G}_{y}\right)(b)\right]
$$

Therefore,

$$
\begin{aligned}
& \Psi\left(\frac{a+b}{2}, \frac{c+d}{2}\right)\left[\begin{array}{c}
\int_{a}^{b}(x-a)^{v_{1}-1} \mathcal{E}_{1}\left(\omega_{1}^{\prime}(x-a)^{\mu_{1}}\right) t \mathcal{G}(x, y) \mathrm{d} x \\
+\int_{a}^{b}(b-x)^{\nu_{1}-1} \mathcal{E}_{1}\left(\omega_{1}^{\prime}(b-x)^{\mu_{1}}\right) t \mathcal{G}(x, y) \mathrm{d} x
\end{array}\right] \\
& \leq\left[\begin{array}{c}
\int_{a}^{b}(x-a)^{v_{1}-1} \mathcal{E}_{1}\left(\omega_{1}^{\prime}(x-a)^{\mu_{1}}\right) \Psi\left(x, \frac{c+d}{2}\right) t \mathcal{G}(x, y) \mathrm{d} x \\
+\int_{a}^{b}(b-x)^{v_{1}-1} \mathcal{E}_{1}\left(\omega_{1}^{\prime}(b-x)^{\mu_{1}}\right) \Psi\left(x, \frac{c+d}{2}\right) t \mathcal{G}(x, y) \mathrm{d} x
\end{array}\right] .
\end{aligned}
$$

Multiplying both sides of (37) by $\mathcal{E}_{2}\left(\omega_{2}^{\prime}(d-y)^{\mu_{2}}\right)(d-y)^{\nu_{2}-1}$ and $\mathcal{E}_{2}\left(\omega_{2}^{\prime}(y-c)^{\mu_{2}}\right)(y-c)^{\nu_{2}-1}$ and integrating with respect to $y$ over $[c, d]$, respectively, we have

$$
\begin{aligned}
& \Psi\left(\frac{a+b}{2}, \frac{c+d}{2}\right)\left[\begin{array}{c}
\int_{a}^{b} \int_{c}^{d}(x-a)^{\nu_{1}-1}(d-y)^{\nu_{2}-1} \mathcal{E}_{1}\left(\omega_{1}^{\prime}(x-a)^{\mu_{1}}\right) \mathcal{E}_{2}\left(\omega_{2}^{\prime}(d-y)^{\mu_{2}}\right) \\
\cdot t \mathcal{G}(x, y) \mathrm{d} y \mathrm{~d} x \\
+\int_{a}^{b} \int_{c}^{d}(b-x)^{\nu_{1}-1}(d-y)^{\nu_{2}-1} \mathcal{E}_{1}\left(\omega_{1}^{\prime}(b-x)^{\mu_{1}}\right) \mathcal{E}_{2}\left(\omega_{2}^{\prime}(d-y)^{\mu_{2}}\right) \\
\cdot t \mathcal{G}(x, y) \mathrm{d} y \mathrm{~d} x
\end{array}\right] \\
& \leq\left[\begin{array}{c}
\int_{a}^{b} \int_{c}^{d}(x-a)^{v_{1}-1}(d-y)^{\nu_{2}-1} \mathcal{E}_{1}\left(\omega_{1}^{\prime}(x-a)^{\mu_{1}}\right) \mathcal{E}_{2}\left(\omega_{2}^{\prime}(d-y)^{\mu_{2}}\right) \\
\cdot \Psi\left(x, \frac{c+d}{2}\right) t \mathcal{G}(x, y) \mathrm{d} y \mathrm{~d} x \\
+\int_{a}^{b} \int_{c}^{d}(b-x)^{v_{1}-1}(d-y)^{\nu_{2}-1} \mathcal{E}_{1}\left(\omega_{1}^{\prime}(b-x)^{\mu_{1}}\right) \mathcal{E}_{2}\left(\omega_{2}^{\prime}(d-y)^{\mu_{2}}\right) \\
\cdot \Psi\left(x, \frac{c+d}{2}\right) t \mathcal{G}(x, y) \mathrm{d} y \mathrm{~d} x
\end{array}\right] \\
& \Psi\left(\frac{a+b}{2}, \frac{c+d}{2}\right)\left[\begin{array}{c}
\int_{a}^{b} \int_{c}^{d}(x-a)^{\nu_{1}-1}(y-c)^{\nu_{2}-1} \mathcal{E}_{1}\left(\omega_{1}^{\prime}(x-a)^{\mu_{1}}\right) \mathcal{E}_{2}\left(\omega_{2}^{\prime}(y-c)^{\mu_{2}}\right) \\
\cdot t \mathcal{G}(x, y) \mathrm{d} y \mathrm{~d} x \\
+\int_{a}^{b} \int_{c}^{d}(b-x)^{\nu_{1}-1}(y-c)^{\nu_{2}-1} \mathcal{E}_{1}\left(\omega_{1}^{\prime}(b-x)^{\mu_{1}}\right) \mathcal{E}_{2}\left(\omega_{2}^{\prime}(y-c)^{\mu_{2}}\right) \\
\cdot t \mathcal{G}(x, y) \mathrm{d} y \mathrm{~d} x
\end{array}\right] \\
& \leq\left[\begin{array}{c}
\int_{a}^{b} \int_{c}^{d}(x-a)^{v_{1}-1}(y-c)^{v_{2}-1} \mathcal{E}_{1}\left(\omega_{1}^{\prime}(x-a)^{\mu_{1}}\right) \mathcal{E}_{2}\left(\omega_{2}^{\prime}(y-c)^{\mu_{2}}\right) \\
\cdot \Psi\left(x, \frac{c+d}{2}\right) t \mathcal{G}(x, y) \mathrm{d} y \mathrm{~d} x \\
+\int_{a}^{b} \int_{c}^{d}(b-x)^{v_{1}-1}(y-c)^{v_{2}-1} \mathcal{E}_{1}\left(\omega_{1}^{\prime}(b-x)^{\mu_{1}}\right) \mathcal{E}_{2}\left(\omega_{2}^{\prime}(y-c)^{\mu_{2}}\right) \\
\cdot \Psi\left(x, \frac{c+d}{2}\right) t \mathcal{G}(x, y) \mathrm{d} y \mathrm{~d} x
\end{array}\right]
\end{aligned}
$$

The same way, we apply Lemma 3 for the functions $F_{2}:[c, d] \rightarrow \mathbb{R}, F_{1}(x)=\Psi\left(\frac{a+b}{2}, y\right)$ and $t \mathcal{G}_{x}:[a, b] \rightarrow \mathbb{R}, t \mathcal{G}_{x}(y)=t \mathcal{G}(x, y)$, and we get:

$$
\begin{gathered}
\Psi\left(\frac{a+b}{2}, \frac{c+d}{2}\right)\left[\begin{array}{c}
\int_{a}^{b} \int_{c}^{d}(x-a)^{v_{1}-1}(y-c)^{v_{2}-1} \mathcal{E}_{1}\left(\omega_{1}^{\prime}(x-a)^{\mu_{1}}\right) \mathcal{E}_{2}\left(\omega_{2}^{\prime}(y-c)^{\mu_{2}}\right) \\
\cdot t \mathcal{G}(x, y) \mathrm{d} y \mathrm{~d} x \\
+\int_{a}^{b} \int_{c}^{d}(x-a)^{v_{1}-1}(d-y)^{v_{2}-1} \mathcal{E}_{1}\left(\omega_{1}^{\prime}(x-a)^{\mu_{1}}\right) \mathcal{E}_{2}\left(\omega_{2}^{\prime}(d-y)^{\mu_{2}}\right) \\
\cdot t \mathcal{G}(x, y) \mathrm{d} y \mathrm{~d} x
\end{array}\right] \\
\leq\left[\begin{array}{c}
\int_{a}^{b} \int_{c}^{d}(x-a)^{v_{1}-1}(y-c)^{\nu_{2}-1} \mathcal{E}_{1}\left(\omega_{1}^{\prime}(x-a)^{\mu_{1}}\right) \mathcal{E}_{2}\left(\omega_{2}^{\prime}(y-c)^{\mu_{2}}\right) \\
\cdot \Psi\left(\frac{a+b}{2}, y\right) t \mathcal{G}(x, y) \mathrm{d} y \mathrm{~d} x \\
+\int_{a}^{b} \int_{c}^{d}(x-a)^{v_{1}-1}(d-y)^{v_{2}-1} \mathcal{E}_{1}\left(\omega_{1}^{\prime}(x-a)^{\mu_{1}}\right) \mathcal{E}_{2}\left(\omega_{2}^{\prime}(d-y)^{\mu_{2}}\right) \\
\cdot \Psi\left(\frac{a+b}{2}, y\right) t \mathcal{G}(x, y) \mathrm{d} y \mathrm{~d} x
\end{array}\right]
\end{gathered}
$$




$$
\begin{gathered}
\Psi\left(\frac{a+b}{2}, \frac{c+d}{2}\right)\left[\begin{array}{c}
\int_{a}^{b} \int_{c}^{d}(b-x)^{v_{1}-1}(y-c)^{\nu_{2}-1} \mathcal{E}_{1}\left(\omega_{1}^{\prime}(b-x)^{\mu_{1}}\right) \mathcal{E}_{2}\left(\omega_{2}^{\prime}(y-c)^{\mu_{2}}\right) \\
\cdot t \mathcal{G}(x, y) \mathrm{d} y \mathrm{~d} x \\
+\int_{a}^{b} \int_{c}^{d}(b-x)^{\nu_{1}-1}(d-y)^{\nu_{2}-1} \mathcal{E}_{1}\left(\omega_{1}^{\prime}(b-x)^{\mu_{1}}\right) \mathcal{E}_{2}\left(\omega_{2}^{\prime}(d-y)^{\mu_{2}}\right) \\
\cdot t \mathcal{G}(x, y) \mathrm{d} y \mathrm{~d} x
\end{array}\right] \\
\leq\left[\begin{array}{c}
\int_{a}^{b} \int_{c}^{d}(b-x)^{v_{1}-1}(y-c)^{\nu_{2}-1} \mathcal{E}_{1}\left(\omega_{1}^{\prime}(b-x)^{\mu_{1}}\right) \mathcal{E}_{2}\left(\omega_{2}^{\prime}(y-c)^{\mu_{2}}\right) \\
\cdot \Psi\left(\frac{a+b}{2}, y\right) t \mathcal{G}(x, y) \mathrm{d} y \mathrm{~d} x \\
+\int_{a}^{b} \int_{c}^{d}(b-x)^{v_{1}-1}(d-y)^{\nu_{2}-1} \mathcal{E}_{1}\left(\omega_{1}^{\prime}(b-x)^{\mu_{1}}\right) \mathcal{E}_{2}\left(\omega_{2}^{\prime}(d-y)^{\mu_{2}}\right) \\
\cdot \Psi\left(\frac{a+b}{2}, y\right) t \mathcal{G}(x, y) \mathrm{d} y \mathrm{~d} x
\end{array}\right] .
\end{gathered}
$$

Adding (38)-(41), we get the first inequality of (31).

For the last inequality of (31), we apply Lemma 3 to the functions

1. $\quad F_{3}:[a, b] \rightarrow \mathbb{R}, F_{3}(x)=\Psi(x, c)$ and $t \mathcal{G}_{y}:[a, b] \rightarrow \mathbb{R}, t \mathcal{G}_{y}(x)=t \mathcal{G}(x, y)$,

2. $\quad F_{4}:[a, b] \rightarrow \mathbb{R}, F_{4}(x)=\Psi(x, d)$ and $t \mathcal{G}_{y}:[a, b] \rightarrow \mathbb{R}, t \mathcal{G}_{y}(x)=t \mathcal{G}(x, y)$,

3. $F_{5}:[c, d] \rightarrow \mathbb{R}, F_{5}(x)=\Psi(a, y)$ and $t \mathcal{G}_{x}:[c, d] \rightarrow \mathbb{R}, t \mathcal{G}_{x}(y)=t \mathcal{G}(x, y)$

4. $\quad F_{6}:[c, d] \rightarrow \mathbb{R}, F_{6}(x)=\Psi(b, y)$ and $t \mathcal{G}_{x}:[c, d] \rightarrow \mathbb{R}, t \mathcal{G}_{x}(y)=t \mathcal{G}(x, y)$,

and we obtain

$$
\begin{aligned}
& \left(\varepsilon_{b^{-}} F_{3} t \mathcal{G}_{y}\right)(a)+\left(\varepsilon_{a^{+}} F_{3} t \mathcal{G}_{y}\right)(b) \leq \frac{F_{3}(a)+F_{3}(b)}{2}\left[\left(\varepsilon_{b^{-}} t \mathcal{G}_{y}\right)(a)+\left(\varepsilon_{a^{+}} t \mathcal{G}_{y}\right)(b)\right], \\
& \left(\varepsilon_{b^{-}} F_{4} t \mathcal{G}_{y}\right)(a)+\left(\varepsilon_{a^{+}} F_{4} t \mathcal{G}_{y}\right)(b) \leq \frac{F_{4}(a)+F_{4}(b)}{2}\left[\left(\varepsilon_{b^{-}} t \mathcal{G}_{y}\right)(a)+\left(\varepsilon_{a^{+}} t \mathcal{G}_{y}\right)(b)\right], \\
& \left(\varepsilon_{d^{-}} F_{5} t \mathcal{G}_{x}\right)(c)+\left(\varepsilon_{c^{+}} F_{5} t \mathcal{G}_{x}\right)(d) \leq \frac{F_{5}(c)+F_{5}(d)}{2}\left[\left(\varepsilon_{d^{-}} t \mathcal{G}_{x}\right)(c)+\left(\varepsilon_{c^{+}} t \mathcal{G}_{x}\right)(d)\right],
\end{aligned}
$$

and

$$
\left(\varepsilon_{d^{-}} F_{6} t \mathcal{G}_{x}\right)(c)+\left(\varepsilon_{c^{+}} F_{6} t \mathcal{G}_{x}\right)(d) \leq \frac{F_{6}(c)+F_{6}(d)}{2}\left[\left(\varepsilon_{d^{-}} t \mathcal{G}_{x}\right)(c)+\left(\varepsilon_{c^{+}} t \mathcal{G}_{x}\right)(d)\right]
$$

Then we have inequality

1. (42) and (43) by $(y-c)^{\nu_{2}-1} \mathcal{E}_{2}\left(\omega_{2}^{\prime}(y-c)^{\mu_{2}}\right)$, respectively $(d-y)^{\nu_{2}-1} \mathcal{E}_{2}\left(\omega_{2}^{\prime}(d-y)^{\mu_{2}}\right)$ and integrating with respect to $y$ over $[c, d]$,

2. (44) and (45) by $(x-a)^{\nu_{1}-1} \mathcal{E}_{1}\left(\omega_{1}^{\prime}(x-a)^{\mu_{1}}\right)$, respectively $(b-x)^{\nu_{1}-1} \mathcal{E}_{1}\left(\omega_{1}^{\prime}(b-x)^{\mu_{1}}\right)$ and integrating with respect to $x$ over $[a, b]$.

We get four inequalities that we are adding, and taking into account the symmetry of $t \mathcal{G}$, we obtain the last inequality of (31). The proof is complete.

Remark 4. If in Theorem 3 we put $\omega=(0,0)$, we obtain [20] (Theorem 8).

\section{Conclusions}

First, we introduced and studied generalized fractional integral operators containing the new extended general Mittag-Leffler function of two variables. We then obtained several new two-dimensional versions of trapezium-like inequalities via coordinated convex functions. We also discussed the linkage of the obtained results with previously known results by considering some special cases. It is expected that the ideas and techniques of this paper may stimulate further research.

Author Contributions: All authors worked jointly and contributed equally. 
Funding: This research received no external funding.

Acknowledgments: Authors are grateful to the editor and anonymous referees for their valuable suggestions. The research is supported by HEC project No. 8081/Punjab/NRPU/R\&D/HEC/2017.

Conflicts of Interest: The authors declare no conflict of interest.

\section{References}

1. Bakula, M.K.; Pećarixcx, J. On the Jensen's inequality for convex functions on the co-ordinates in a rectangle from the plane. Taiwan. J. Math. 2006, 5, 1271-1292. [CrossRef]

2. Dragomir, S.S. On Hadamard's inequality for convex functions on the co-ordinates in a rectangle from the plane. Taiwan. J. Math. 2001, 5, 775-788. [CrossRef]

3. Latif, M.A.; Alomari, M. Hadamard-type inequalities for product two convex functions on the co-ordinates. Int. Math. Forum 2009, 4, 1645-1656.

4. Özdemir, M.E.; Akdemir, A.; Ekinci, A. New integral inequalities for co-ordinated convex functions. RGMIA Res. Rep. Collect. 2012, 15, 15.

5. Sarikaya, M.Z.; Set, E.; Özdemir, M.E.; Dragomir, S.S. New some Hadamard's type inequalities for co-ordinated convex functions. Tamsui Oxf. J. Math. Sci. 2012, 28, 137-152.

6. Gorenflo, R.; Mainardi, F. Fractional Calculus: Integral and Differential Equations of Fractional Order; Springer: Wien, Ausria, 1997; pp. 223-276.

7. Salim, T.O.; Faraj, A.W. A generalization of Mittag-Leffler function and integral operator associated with fractional calculus. J. Fract. Appl. 2012, 3, 1-13.

8. Srivastava, H.M.; Tomovski, Z̆. Fractional calculus with an integral operator containing generalized Mittag-Leffler function in the kernel. Appl. Math. Comput. 2009, 211, 198-210. [CrossRef]

9. Prabhakar, T.R. A singular integral equation with a generalized Mittag-Leffler function in the kernal. Yokohama Math. J. 1971, 19, 7-15.

10. Gorenflo, R.; Kilbas, A.A.; Mainardi, F.; Rogosin, S.V. Mittag-Leffler Functions, Related Topics and Applications; Springer: Berlin, Germany, 2014; Volume 2.

11. Abbas, G.; Khan, K.A.; Farid, G.; Rehman, A.-U. Generalizations of some fractional integral inequalities via generalized Mittag-Leffler function. J. Inequal. Appl. 2017, 2017, 121. [CrossRef] [PubMed]

12. Kunt, M.; İşcan, İ.; Yazici, N.; Gözütok, U. On new inequalities of Hermite-Hadamard-Fejér type for harmonically convex functions via fractional integrals. SpringerPlus 2016, 5, 635. [CrossRef] [PubMed]

13. Mihai, M.V. Some Hermite-Hadamard type inequalities obtained via Riemann-Liouville fractional calculus. Tamkang J. Math. 2013, 44, 411-416. [CrossRef]

14. Mihai, M.V. New inequalities for co-ordinated convex functions via Riemann-Liouville fractional calculus. Tamkang J. Math. 2014, 45, 285-296.

15. Mihai, M.V.; Mitroi, F.C. Hermite-Hadamard type inequalities obtained via Riemann-Liouville fractional calculus. Acta Math. Comen. 2014, 83, 209-2015.

16. Nisan, K.S.; Rahman, G.; Băleanu, D.; Mubeen, S.; Arshad, M. The (k,s)-fractional calculus of k-Mittag-Leffler function. Adv. Differ. Equ. 2017, 118. [CrossRef]

17. Noor, M.A.; Mihai, M.V.; Noor, K.I.; Awan, M.U. Fractional Hermite-Hadamard inequalities for differentiable s-Godunova-Levin functions. Filomat 2016, 30, 3235-3241.

18. Sarikaya, M.Z.; Yildirim, H. On Hermite-Hadamard type inequalities for Riemann-Liouville fractional integrals. Miskolc Math. Notes 2016, 17, 1049-1059. [CrossRef]

19. İşcan, İ. Hermite-Hadamard-Fejér type inequalities for convex functions via fractional integrals. Stud. Univ. Babeş-Bolyai Math. 2015, 60, 355-366.

20. Yaldiz, H.; Sarikaya, M.Z.; Dahmani, Z. On the Hermite-Hadamard-Fejér-type inequalities for co-ordinated convex functions via fractional integrals. Int. J. Optim. Control Theor. Appl. 2017, 7, 205-215. [CrossRef] 